IMA Journal of Applied Mathematics (2018) Page 1 of 27 doi:10.1093/imamat/xxx000

\title{
The influence of distributed chemical reaction groups in a multiphase coffee bean roasting model
}

\author{
Nabil T. Fadai ${ }^{a *}$, Zahra Akram ${ }^{b}$, Fabien Guilmineau $^{b}$, John Melrose $^{b, c}$, Colin \\ P. PleAse ${ }^{a}$ AND Robert A. VAN GordeR ${ }^{a}$ \\ ${ }^{a}$ Mathematical Institute, University of Oxford, Andrew Wiles Building, Radcliffe Observatory \\ Quarter, Woodstock Road, Oxford OX2 6GG United Kingdom \\ ${ }^{b}$ Jacobs Douwe Egberts R\&D UK Ltd, Ruscote Avenue, Banbury OX16 2QU United Kingdom \\ ${ }^{c}$ Koninklijke Douwe Egberts B.V., Oosterdoksstraat 80, 1011 DK Amsterdam Netherlands
}

[Received on 10 May 2018]

\begin{abstract}
The coffee industry relies on fundamental research to improve the techniques and processes related to its products. While recent theoretical and modelling work has focused on the heat and mass transfer processes within roasting coffee beans, modelling and analysis of chemical reactions in the context of multiphase models of roasting beans has not been well-studied. In this paper, we incorporate modified evaporation rates and chemical reaction groups to improve existing mathematical models of roasting coffee beans. We model the phase change from liquid to vapour water within the bean during roasting using first-order Arrhenius-like global reactions, and for other components of the bean, we consider a threecomponent solid phase model which includes sucrose, reducing sugars, and other organic compounds, which allows for porosity of the solid matrix to vary during the roasting process. We non-dimensionalise and then solve the multiphase model numerically, comparing the simulations with data we have collected through full bean and chopped bean experiments. We demonstrate that numerical solutions of the enhanced multiphase model with global water reactions and three-component solid phase reactions agree with experimental data for the average moisture content in whole beans and small chunks of bean, but that the data allows for a range to possible parameter values. We discuss other experimental data that might be collected to more firmly determine the parameters and hence the behaviour more generally. The indeterminacy of the parameters ensures that the additional effects included in the model will enable better understanding the coffee bean roasting process.
\end{abstract}

Keywords:

multiphase coffee bean roasting model, water activity, sorption isotherm, sugar chemical pathway, reaction groups

\section{Introduction}

Coffee is one of the most valuable commodities in the world (Talbot, 2014) and the coffee industry relies on fundamental research to improve the techniques and processes related to its products. While experimental data is present in the literature (see e.g. Baggenstoss (2008); Schenker (2000); Wang and Lim (2014)), few theoretical techniques, other than regression analyses and empirical models, are used to analyse these data. Two notable exceptions are Fabbri et al. (2011), where the authors present a

*Email: Nabil.Fadai@maths.ox.ac.uk

(c) The author 2018. Published by Oxford University Press on behalf of the Institute of Mathematics and its Applications. All rights reserved. 
coupled moisture-temperature mathematical model, and Fadai et al. (2017, 2018), where multiphase modelling and the transport of water and water vapour are emphasized. While these models serve as a good starting point to modelling the roasting of coffee beans, they are inadequate in explaining some experimental observations and need to be extended and revised.

In Fadai et al. (2017), a multiphase model was derived describing the roasting of a single coffee bean. From this, a simplified multiphase model was created to model the transport of heat, water, and water vapour. This was seen as an improvement to the model proposed in Fabbri et al. (2011), which focused on a one-phase quantity for moisture while not distinguishing whether the moisture was in liquid or gas form. Additionally, the model in Fabbri et al. (2011) relies on average moisture transport rather than local moisture transport, while the multiphase model derived in Fadai et al. (2017) distinguishes water into liquid and gas phases and only uses local mechanisms. Despite these improvements, there are many practical ways that the multiphase model proposed in Fadai et al. (2017) might be refined. First, the model only accounts for variations in temperature, water content, and water vapour pressure. In reality, porosity will also vary and the effect of various other gases (such as $\mathrm{CO}_{2}$ and air) should be accounted for. Additionally, this simplified model does not address the myriad of chemical reactions that occur within the coffee bean as it is roasted. Finally, as we will demonstrate in this paper, the simplified model in Fadai et al. (2017) does not replicate experimental data when size of the coffee bean is altered.

In this paper, we shall extend the coffee bean roasting model of Fadai et al. (2017) in a number of ways. Motivated in part by literature on timber drying (e.g. Perré and Turner (1996)) we consider more detailed mechanisms for the evaporation rate within a coffee bean. Specifically, we view evaporation as a multistep process including accounting for a distribution of chemical reactions. Furthermore, we incorporate water activity (Krupińska et al. (2007); Oswin (1946)) into our evaporation model to account for how water changes phase from liquid to vapour within a coffee bean structure. To account for the many chemical reactions present within the roasting bean, we also include a simplified sugar chemical pathway model representative of key reaction groups that occur during the roasting process. Finally, a spherical "shell" geometry is used to provide a more realistic representation of the geometry of a coffee bean. The resulting model is then non-dimensionalised and solved numerically.

The remainder of this paper is organized as follows. In Section 2, we discuss some limitations of the existing multiphase models for coffee bean roasting, pointing out that experiments we conducted show disagreement with existing models when coffee beans are chopped into smaller volumes prior to roasting. In Section 3, we model the phase change from liquid to vapour water within the bean during roasting, accounting for a multi-step process and assuming that the phase changes can be modelled using various first-order Arrhenius-like reactions. In Section 4, we propose a three-component solid phase model - the Sugar Pathway Model - which includes sucrose, reducing sugars, and other organic compounds. We include chemical reactions in the solid phase to alter the porosity. Furthermore, in order to more realistically represent the bean geometry, we derive an effective length scale for the model. In Section 5, we non-dimensionalise the multiphase model and give the relevant parameter groups, while in Section 6, we solve this model numerically and compare the resulting solutions with data we obtained from roasting experiments we have performed. Finally, in Section 7, we summarise and discuss the results.

\section{Fitting existing multiphase models to experimental data}

In Fadai et al. (2017), mechanisms determining the local moisture content were different from the onephase model of Fabbri et al. (2011). The predicted average moisture content in the bean agreed well 
with previous model as well as previously existing experimental data in Fabbri et al. (2011). However, there are certain parameters in these models that were chosen to fit the experimental data presented. Therefore, it is important to validate these parameter values, by means of other experimental data, to determine the robustness of the model. We first discuss the validity of the parameters compared to values used elsewhere in the literature and then present new experimental data for different bean configurations. One critisism of the model in Fadai et al. (2017) was that the gas permeability $k_{g}$, needed to match existing experimental data, was approximately $2 \times 10^{-19} \mathrm{~m}^{-2}$. This is far smaller than the values $10^{-15}-10^{-12} \mathrm{~m}^{-2}$ used for typical organic materials, such as wood (e.g. Comstock (1970)). We will discuss how the model in Fadai et al. (2017) might be modified to make such fitted parameter values physically realistic.

\subsection{The chopped green coffee bean experiment}

To further validate the model parameter values, we undertook the chopped green coffee bean experiment proposed in Fadai et al. (2017). Specifically, this experiment explores the question: if we coarsely break up green coffee beans prior to roasting, how much faster does the water vapour leave the bean? To examine this behaviour, washed Arabica Peru coffee beans were ground on a NETZSCH Condux mill set with the widest possible gap settings between rotor and stator. The ground beans obtained were sieved through 2 metal mesh sieves placed above each other, with mesh sizes of $2.5 \mathrm{~mm}$ and $1.4 \mathrm{~mm}$ respectively. Hence, we can infer that the radius of a coffee bean chunk that we consider is between $1.3 \mathrm{~mm}$ and $0.7 \mathrm{~mm}$ and we will typically consider it to have radius $0.7 \mathrm{~mm}$.

Roasting experiments were carried out on a Probat BRZ2 electric drum laboratory roaster in batches of $100 \mathrm{~g}$ and roasted at approximately $230^{\circ} \mathrm{C}$ for $180-300$ seconds. The product temperature, measured by the fixed probe built in the roaster drum, was recorded throughout the roast. The batch of chopped beans was removed at regular intervals during roasting, cooled in ambient air and immediately placed in a sealed container. Residual bean moisture was measured gravimetrically: a $10 \mathrm{~g}$ sample of roasted whole beans was placed in an oven set at $103-105{ }^{\circ} \mathrm{C}$ for 16 hours and allowed to cool down in a desiccator. To provide a control for comparison, whole green beans of approximately $4 \mathrm{~mm}$ average radius were also roasted at $230^{\circ} \mathrm{C}$ and their moisture content recorded at regular intervals (see Table 1).

As these bean chunks look roughly spherical, we consider predicting their drying behaviour using the Multiphase Model shown in Fadai et al. (2017) on a spherical geometry with radius $L=0.7 \mathrm{~mm}$ while keeping all other parameter values unchanged. Results of this experiment are shown Figure 1, where we observe that the moisture loss predicted by the Multiphase Model significantly disagrees with the experimental data. We might expect that because the behaviour is primarily determined by a diffusive process, the effective length scale of the model has been decreased by a factor of $\frac{0.7}{4}$, the drying time of the bean chunk will be $\left(\frac{0.7}{4}\right)^{2} \approx 3.1 \%$ of the time for a whole bean. However, the data in Figure 1 shows that, when drying chunks, they take roughly $65 \%$ of the whole bean drying time. Hence, this simple model with the parameters chosen to fit whole bean behaviour does not translate well with a different length scale.

\section{Evaporation mechanisms in roasting coffee beans}

To gain a better fit between the model predictions and experimental data over a wide range of conditions, the model in Fadai et al. (2017) needs to be extended. Motivated by the existing literature on timber drying (e.g. Perré and Turner (1996)), the model of evaporation was seen as the critical element to 


\begin{tabular}{c|c|c} 
Time, s & Moisture Content (Whole Bean), \% & Moisture Content (Chopped Bean), \% \\
\hline 0 & 10.2 & 10.2 \\
60 & 9.05 & 6.37 \\
120 & 7.13 & 3.18 \\
180 & 5.72 & 1.45 \\
210 & 5.07 & 1.22 \\
240 & 2.27 & - \\
270 & 1.62 & -
\end{tabular}

Table 1. Moisture Content of whole coffee beans and chopped coffee beans during a $230^{\circ} \mathrm{C}$ roast.

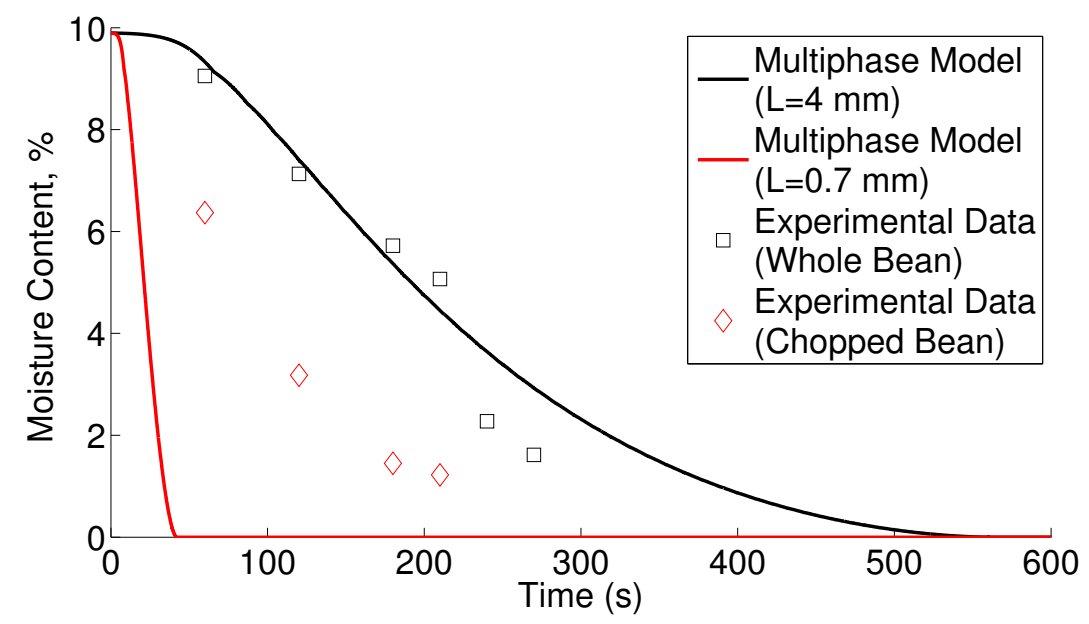

FIG. 1. Comparison of the average moisture content in a bean chunk versus a whole bean during a $230^{\circ} \mathrm{C}$ roast. The solid lines correspond to the average moisture loss determined by the simplified multiphase model presented in Fadai et al. (2017), and the markers correspond to experimental data seen in Table 1. Aside from the effective radius $L$ and roast temperature $T_{\infty}=230^{\circ} \mathrm{C}$, all other parameters are the same as those used in Fadai et al. (2017). 
describe accurately. A simplistic view of the evaporation process is the following. Firstly, there is water in the cells which is not accessible until the cell structure degrades (called the "yellowing stage" in roasting). When the cell degrades, this cell water joins the water already bound in the cellular matrix of the bean. This bound water then slowly evaporates to become water vapour. How quickly it evaporates is determined by the strength of the chemical bond between the water and the matrix, as well as the surrounding water vapour pressure. One of the difficulties in deriving such a model is that there are a myriad of possible components to be degraded in cells and a similar large number of compounds that water might bind to. The final model has a few parameters in it and we shall present ideas from pyrolysis of coal, the Distributed Activation Energy Method (DAEM) discussed in Please et al. (2003), to indicate how these parameters might be connected to independently measurable quantities. Hence, the resulting modelling of evaporation replaces the simple Langmuir evaporation equation given in Fadai et al. (2017).

\subsection{The sorption isotherm}

We start by considering how water bound to the matrix might evaporate, since this mechanism was in Fadai et al. (2017), where it was assumed that there was no binding and so the evaporation was described by a simple Langmuir expression for pure water. To account for binding, we need to consider the water activity $a_{w}$ associated with each type of binding site. The site will either fill or empty, depending on the surrounding vapour pressure $p_{v}$ and the property that determines this direction of flow is the equilibrium vapour pressure of the site $p_{v}^{*}$, which is given by

$$
p_{v}^{*}=a_{w} p_{S T}(T),
$$

where $p_{S T}(T)$ is the pure steam table pressure as given, for example, in Dean (1999):

$$
p_{S T}(T)=A_{1} \exp \left(A_{2}-A_{3} / T\right),
$$

with $A_{1}, A_{2}$, and $A_{3}$ being constants. The binding site will fill if the vapour pressure is too high (i.e. $\left.p_{v}>p_{v}^{*}\right)$ and empty otherwise. This relationship is normally prescribed by the sorption isotherm; in the literature (see e.g. Krupińska et al. (2007); Oswin (1946)), the sorption isotherm relates the mass fraction of water to dry solids, $X$, to the water activity $a_{w}$.

In terms of variables presented here, we relate the bound water mass fractions to their volume fractions using

$$
X=\frac{\frac{\rho_{w}}{\rho_{s}} \phi S}{1-\phi},
$$

where $\rho_{s}$ is the density of the dry coffee solids, $1-\phi$ is the volume fraction occupied by the solid coffee bean, $\phi S$ is the volume fraction occupied by liquid, and $a_{w}(X)$ is defined by

$$
p_{v}^{*}=a_{w}(X) p_{S T}(T),
$$

Many models relating $a_{w}$ to $X$ have been proposed (see for example (Krupińska et al., 2007)), but here we will consider the simple relationship proposed in Oswin (1946):

$$
X=B_{1}\left(\frac{a_{w}}{1-a_{w}}\right)^{B_{2}}
$$




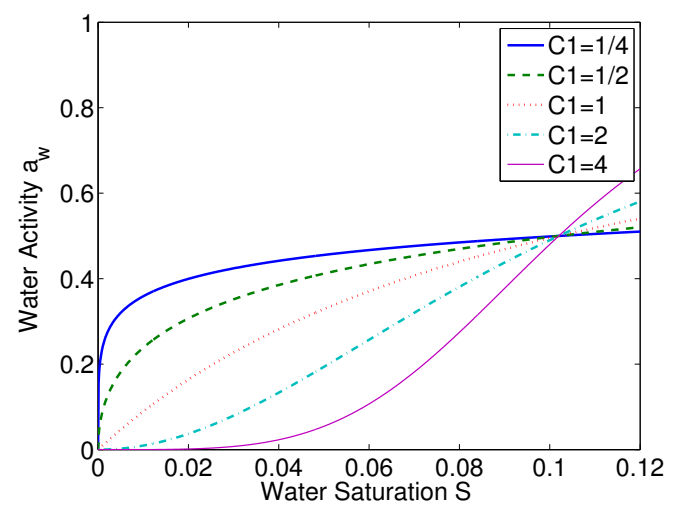

(a)

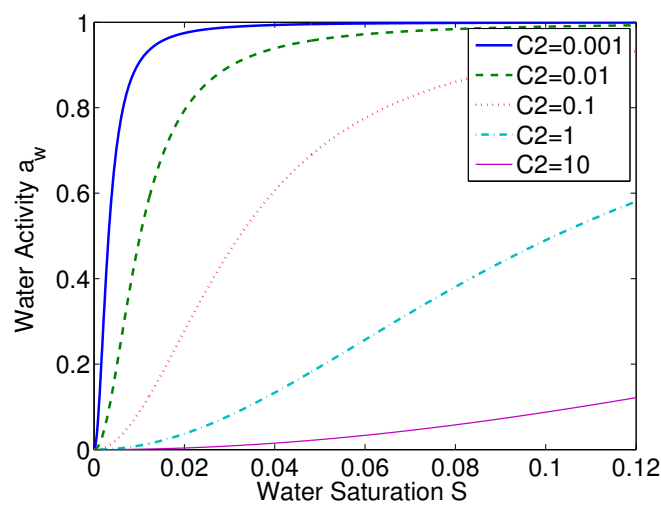

(b)

FIG. 2. Comparison of the water activity function $a_{w}$, defined in (3.6), with $\phi=0.5$ and $\sigma=0.1$. (a) Parameter $C_{1}$ is varied with $C_{2}=1$. (b) Parameter $C_{2}$ is varied with $C_{1}=2$.

implying that

$$
a_{w}=\frac{(\phi S)^{C_{1}}}{(\phi S)^{C_{1}}+C_{2} \sigma^{C_{1}}(1-\phi)^{C_{1}}},
$$

where $\sigma$ is the initial volumetric liquid-to-void ratio in the coffee bean and $C_{1}$ and $C_{2}$ are constants. It is important to note that the density ratio $\frac{\rho_{w}}{\rho_{s}}$ is absorbed in the fitting parameter $C_{2}$. Therefore, our sorption isotherm $p_{v}^{*}$, relating vapour pressure to water content, can be written as

$$
p_{v}^{*}(T, \phi, S)=\frac{P_{0} D_{1}(\phi S)^{C_{1}} \exp \left[-\frac{D_{2} T_{0}}{T_{\infty}-T_{0}}\left(\frac{T_{\infty}}{T}-1\right)\right]}{(\phi S)^{C_{1}}+C_{2} \sigma^{C_{1}}(1-\phi)^{C_{1}}},
$$

where $D_{1}=\frac{A_{1}}{P_{0}} \exp \left(A_{2}-\frac{A_{3}}{T_{\infty}}\right)$ and $D_{2}=\frac{A_{3}\left(T_{\infty}-T_{0}\right)}{T_{0} T_{\infty}}$. Figure 2 shows how $a_{w}(S)$ varies with different values of $C_{1}$ and $C_{2}$ for fixed porosity. Comparing the model developed here with that used used in Fadai et al. (2017), we note that the previous sorption isotherm is independent of volumetric fractions (e.g. $C_{2}=0$ ). The literature on timber drying (see e.g. Perré and Turner (1996)) uses a similar approach, but assumes that the reaction is so rapid that the sorption isotherm is equal to the water vapour pressure. We also assume that this reaction is rapid, but will state the underlying governing conservation equations for completeness.

3.1.1 The incorporation of $p_{v}^{*}$ into the Multiphase Model It is natural at this stage to compare the original Multiphase Model shown in Fadai et al. (2017) with one incorporating the sorption isotherm. By changing $p_{S T}(T)$ in Fadai et al. (2017) with $p_{v}^{*}(T, \phi, S)$ shown in (3.7), we can solve this modified Multiphase Model with the same parameters as used in Fadai et al. (2017), with $C_{1}$ and $C_{2}$ being given in Corrêa et al. (2010) and $T_{\infty}=230^{\circ} \mathrm{C}$. With reference to Figure 3, there are two main differences between these two models. Firstly, the moisture content decays significantly faster in the original Multiphase Model (without the sorption isotherm) than in the modified Multiphase Model with the sorption isotherm. Secondly, we note that the maximum vapour pressure inside the bean is much smaller in the 


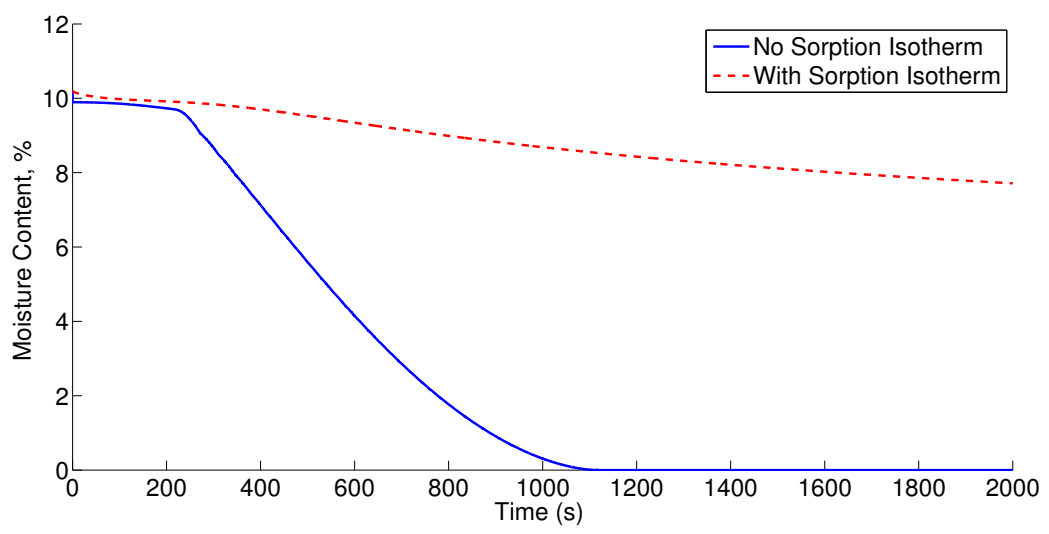

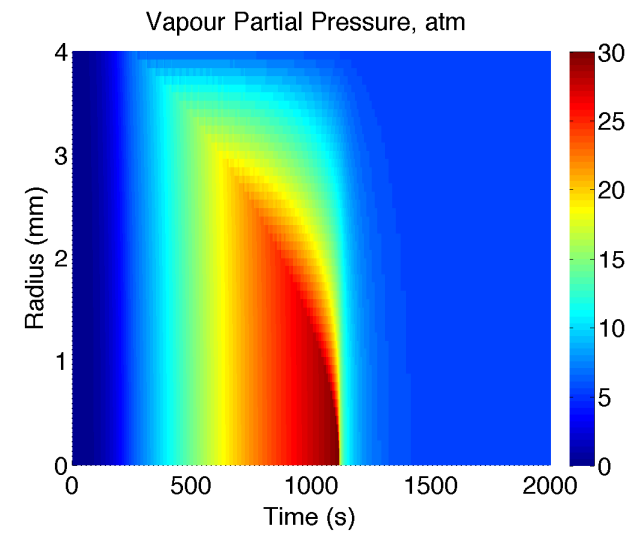

(b)

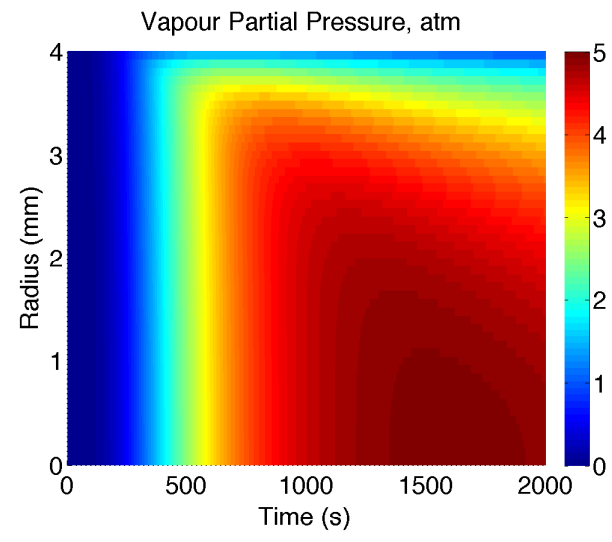

(c)

FIG. 3. (a) Comparison of the moisture loss predicted by the Multiphase Model shown in Fadai et al. (2017) with and without the incorporation of the sorption isotherm defined in (3.7). Bottom: Vapour pressure $p_{v}(r, t)$ as predicted by the Multiphase Model (b) without the sorption isotherm and (c) with the sorption isotherm. All parameters are the same as those used in Fadai et al. (2017), with $C_{1}$ and $C_{2}$ in (3.7) being given in Corrêa et al. (2010) and $T_{\infty}=230^{\circ} \mathrm{C}$.

modified Multiphase Model than in the original Multiphase Model. This is due to the water activity function reducing the maximal vapour pressure for a given moisture content, which in turn slows the rate at which water vapour can be transported to the outside of the bean.

3.1.2 Relating the maximum vapour pressure to evaporation parameters The modified Multiphase Model predicts that the coffee bean will dry at a significantly slower speed than the original model stated in Fadai et al. (2017). However, this could be compensated for by increasing the gas permeability $\left(k_{g}\right)$ to a more realistic value for similar organic compounds, such as wood. Such a change in parameters would cause the maximal vapour pressure observed in the coffee bean, denoted as $p_{v, \max }$, to decrease (as seen in Table 2). With reference to Figure 4, we see that $\log p_{v, \max }$ is linear in $\log k_{g}$ away from zero and has a slope of approximately $-\frac{1}{2}$, which indicates that $p_{v, \max }=O\left(k_{g}^{-1 / 2}\right)$ for $k_{g} \gg 1$. However, we also 


\begin{tabular}{c|c} 
Gas Permeability $k_{g}, \mathrm{~m}^{2}$ & Maximal Vapour pressure $p_{v, \max }$, atm \\
\hline $2 \times 10^{-19}$ & 4.97 \\
$2 \times 10^{-18}$ & 3.77 \\
$2 \times 10^{-17}$ & 1.92 \\
$2 \times 10^{-16}$ & 0.779 \\
$2 \times 10^{-15}$ & 0.280 \\
$2 \times 10^{-14}$ & 0.0940 \\
$2 \times 10^{-13}$ & 0.0304 \\
$2 \times 10^{-12}$ & 0.0100
\end{tabular}

Table 2. Maximum vapour pressure experienced in the modified Multiphase Model for various gas permeabilities.

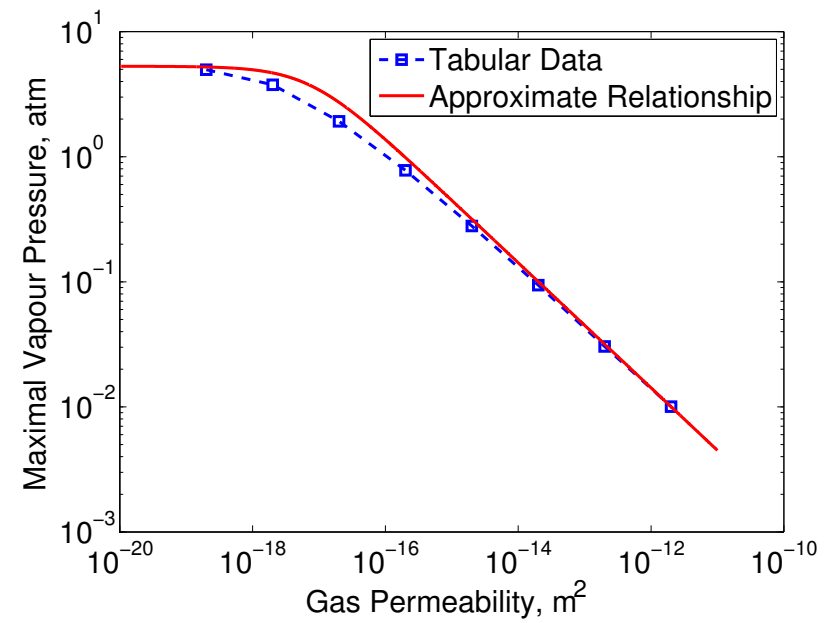

FIG. 4. Log-log plot of $p_{v, \max }$ versus $k_{g}$. The tabular data is shown in Table 2 and the approximate relationship refers to Equation (3.8), with $k_{0} \approx 7.2 \times 10^{-18} \mathrm{~m}^{2}$.

note that $p_{v, \max }$ cannot exceed $p_{v}^{*}\left(T_{\infty}, \phi, \sigma\right)$ if we impose that condensation cannot occur. Therefore, we infer the relationship

$$
p_{v, \max }=\frac{p_{v}^{*}\left(T_{\infty}, \phi, \sigma\right)}{\sqrt{\frac{k_{g}}{k_{0}}+1}},
$$

where $k_{0}$ is chosen to fit the data.

If we take the view that the value of $k_{g}$ should be appropriate for permeabilities in wood, we would choose $k_{0}$ so that the curve intersects the data when $k_{g}=2 \times 10^{-12}$, which implies that $k_{0} \approx 7.2 \times 10^{-18}$ $\mathrm{m}^{2}$. As we can see in Figure 4, this approximate relationship between $p_{v, \max }$ and $k_{g}$ agrees well with the data in the physically relevant parameter range. We note, however, that when using this value of $k_{g}$, the effect of sorption isotherm implies that the maximal vapour pressure, as shown in Table 2, is only a fraction of an atmosphere above the external environment; we discuss the consequences of this later. 


\subsection{Modelling the degradation of cells}

We now consider how to model the degradation of the cells and how the water in the cells then moves into bound water. We will assume that the cell water volume fraction is given by $\phi S-w_{b}-w_{n}$ where $\phi$ is the porosity, $S$ is the water saturation, and $w_{b}+w_{n}$ is the total bound water fraction. The bound water will be separated into $w_{b}$ the water that can be evaporated and $w_{n}$ a fraction that cannot be removed. A simple model of degradation of the cells is to take

$$
\frac{\partial}{\partial t}\left[\phi S-w_{b}-w_{n}\right]=-\left(\phi S-w_{b}-w_{n}\right) \mathscr{R}_{1}(T)
$$

where $\mathscr{R}_{1}(T)$ is the effective rate that cell water becomes bound water due to degradation of the cell. We now discuss how this effective rate might be determined from degradation rates for the many chemical components of a cell.

\subsection{Modelling degradation using a distribution of chemical reactions}

To motivate the framework of a distributed chemical reaction group, we first consider a single first-order reaction $A \rightarrow B$ with reaction rate $\mathscr{K}$, where $A$ is a particular cell component and $B$ is the product of its degradation. If we assume an first-order Arrhenius reaction model (Laidler, 1984), we obtain the system of differential equations

$$
\begin{aligned}
\frac{\partial \phi_{A}}{\partial t} & =-\mathscr{K} \phi_{A} \exp \left(-\frac{E}{R T}\right), \\
\frac{\partial \phi_{B}}{\partial t} & =\frac{\rho_{A}}{\rho_{B}} \mathscr{K} \phi_{A} \exp \left(-\frac{E}{R T}\right),
\end{aligned}
$$

where $\phi_{A}$ and $\phi_{B}$ are the volumetric fractions of chemicals $A$ and $B$ respectively. This is an appropriate form to model reaction kinetics for a single chemical reaction and can readily be extended to many components by writing an ODE for each. However, when modelling a very large group of chemical reactions, it may be appropriate to make a simple model by assuming some distribution of chemical reactions. For simplicity, we will assume that all chemical reactions in the group are independent and that they are first-order governed by Arrhenius-like kinetics. We then model a group of chemical reactions by assuming each component has a different activation energy $E$ and reaction pre-factor $\mathscr{K}$ so that they can be described by a density distribution $f(E)$ over activations energies. This approach is motivated from the DAEM, whose original application was in modelling various chemical reactions in the pyrolysis of coal. Mathematically, this distributed chemical reaction model takes the form of a single reaction for the group with a global reaction rate, $\mathscr{R}(T)$, given by

$$
\mathscr{R}(T)=\int_{0}^{\infty} \mathscr{K}(E) \exp \left(-\frac{E}{R T}\right) f(E) d E .
$$

As a specific example, we can assume that the pre-factors are constant and independent of the activation energy (i.e. $\mathscr{K}(E) \equiv \mathscr{K}$ ) and that the distribution of activation energies of the chemical reactions is Gaussian with a large mean $\mu_{E}$ and standard deviation $\sigma_{E}$ :

$$
f(E)=\frac{1}{\sigma_{E} \sqrt{2 \pi}} \exp \left(-\frac{\left(E-\mu_{E}\right)^{2}}{2 \sigma_{E}^{2}}\right) .
$$


Thus, if we now view the process $a \rightarrow b$ as a group of chemical reactions, we would model this group of reactions as

$$
\begin{aligned}
\frac{\partial \phi_{a}}{\partial t} & =-\phi_{a} \mathscr{R}(T), \\
\frac{\partial \phi_{b}}{\partial t} & =\frac{\rho_{a}}{\rho_{b}} \phi_{a} \mathscr{R}(T),
\end{aligned}
$$

where

$$
\begin{aligned}
\mathscr{R}(T) & =\frac{\mathscr{K}}{\sigma_{E} \sqrt{2 \pi}} \int_{0}^{\infty} \exp \left(-\frac{E}{R T}-\frac{\left(E-\mu_{E}\right)^{2}}{2 \sigma_{E}^{2}}\right) d E \\
& =\frac{\mathscr{K}}{2} \exp \left(\frac{\sigma_{E}^{2}}{2 R^{2} T^{2}}-\frac{\mu_{E}}{R T}\right) \operatorname{erfc}\left(\frac{\sigma_{E}}{\sqrt{2} R T}-\frac{\mu_{E}}{\sqrt{2} \sigma_{E}}\right) .
\end{aligned}
$$

and $\phi_{a}$ and $\phi_{b}$ are the volumetric fractions of chemical groups $a$ and $b$ respectively.

\subsection{Approximation of the global reaction rate}

While the global reaction rate in (3.16) better represents more complex groups of chemical reactions, the explicit formula for $\mathscr{R}(T)$ shown in (3.16) is complicated to work with. We therefore consider a simplified version of $\mathscr{R}(T)$ given by

$$
\mathscr{R}(T) \approx \tilde{\mathscr{R}}(T):=\mathscr{R}\left(T_{\infty}\right) \exp \left[\log \left(\frac{\mathscr{R}\left(T^{*}\right)}{\mathscr{R}\left(T_{\infty}\right)}\right)\left(\frac{T_{\infty}-T}{T_{\infty}-T^{*}}\right)\right],
$$

where $T^{*}$ is chosen so that $\max _{T \in\left[T_{0}, T_{\infty}\right]}|\tilde{\mathscr{R}}(T)-\mathscr{R}(T)|$ is minimized, i.e. the maximal absolute error between the global reaction rate and its approximating function is minimal. This exponential approximation is appropriate as we are in the parameter range where $\mu_{E} \gg \sigma_{E}$, implying that $\mathscr{R}(T)$ is always concave up in the specified temperature range. Equivalently, this exponential behaviour is valid when the Arrhenius dependence can readily be approximated by an exponential, as is valid for most roasting conditions.

As an example, we consider the hydrolysis of surcrose to glucose and fructose, which has parameters $\mu_{E}=109200$ and $\mathscr{K}=2.0 \times 10^{14}$ (Tombari et al., 2007). We anticipate that the standard deviation of this global reaction rate is small, so we choose $\sigma_{E}=1000$. As we can see in Figure 5, the fit exponential approximation agrees well with $\mathscr{R}(T)$ on the temperature range of $20^{\circ} \mathrm{C}-250^{\circ} \mathrm{C}$.

Using these ideas, we assume that the global reaction rate can be approximated by

$$
\mathscr{R}(T) \approx \alpha \exp \left(-\frac{\beta\left(T_{\infty}-T\right)}{T_{\infty}-T_{0}}\right),
$$

where $\alpha>0$ and $\beta>0$ are parameters to be determined experimentally. To help identify a possible realistic range for these parameters, we note that for the sucrose hydrolysis example, when $T_{0}=20^{\circ} \mathrm{C}$ and $T_{\infty}=250^{\circ} \mathrm{C}$, this yields $\alpha \approx 2560 \mathrm{~s}^{-1}, \beta \approx 11.6$.

\subsection{Modelling evaporation of bound water}

The second process for the liquid phase is evaporation of the bound water into water vapour. We denote the volume fraction of bound water that might evaporate by $w_{b}$ and we assume that there is a constant 


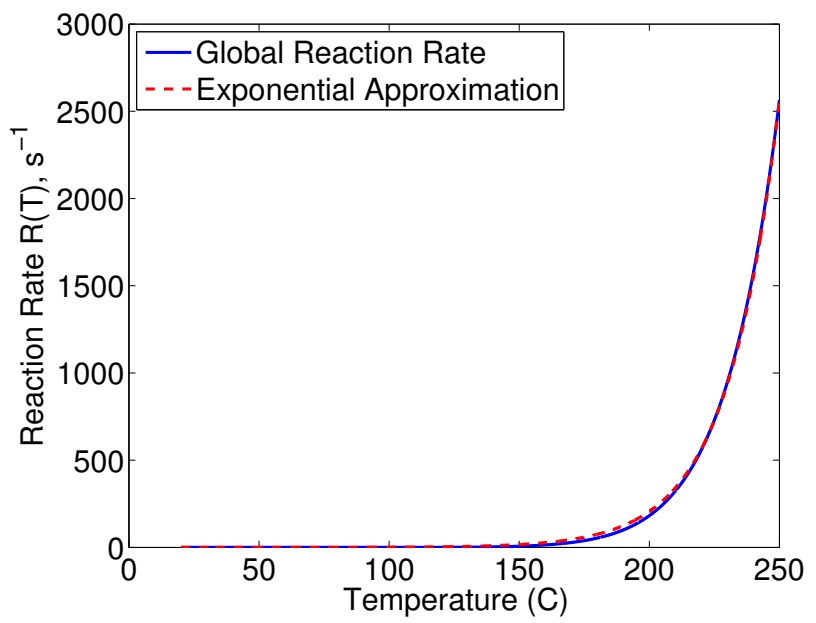

FIG. 5. Comparison of the hydrolysis of sucrose modelled using the global reaction rate $\mathscr{R}(T)$, defined in (3.16), and its exponential approximation $\tilde{\mathscr{R}}(T)$, defined in (3.17).

non-reactive liquid fraction $w_{n}$ that stays bound in the matrix. There are many different components of the bound water, each bound to a different chemical in the matrix. Hence, we might consider there to be a distribution of possible activation energies associated with these bound sites and again use the concepts of the DAEM.

Having described the behaviour of a single type of binding site for the water in Section 3.1, we now need to write down the effective equations when there is a distribution of sites with different activation energies. We find there are two elements that appear in the model when applying DAEM concepts: an effective reaction rate $\mathscr{R}_{2}(T)$ for the evaporation, and the previously mentioned sorption isotherm $p_{v}^{*}$. Using the ideas from Section 3.1, and recalling that bound water is also created from cell water by cell degeneration, the model for bound water becomes

$$
\frac{\partial w_{b}}{\partial t}=\left(\phi S-w_{b}-w_{n}\right) \mathscr{R}_{1}(T)-w_{b}\left[p_{v}-p_{v}^{*}(T, \phi, S)\right] \mathscr{R}_{2}(T) .
$$

We note that by combining (3.19) with (3.9), we can derive the conservation equation for the fraction of the entire liquid phase $\phi S$ :

$$
\frac{\partial}{\partial t}[\phi S]=-w_{b}\left[p_{v}-p_{v}^{*}(T, \phi, S)\right] \mathscr{R}_{2}(T) .
$$

A summary of the parameter values used for the effective reaction rates of degradation and evaporation is shown in Table 3.

\section{A three-component solid phase model (the Sugar Pathway Model)}

It is crucial for understanding coffee roasting to know how sucrose and other sugars in the bean react to produce flavour compounds. In particular, we will model the main sugar reactions via a simplified chemical reaction pathway that is motivated by the reactions proposed by van Boekel (2006). To account for important effects of these reactions, we will also need to incorporate variable porosity, additional 
gas species, and some effective thermal properties in the model. With appropriate boundary and initial conditions, these conservation equations will be referred to as the Sugar Pathway Model.

\subsection{A simplified sugar pathway and the inclusion of variable porosity}

For simplicity, and motivated by the reactions described in van Boekel (2006), we consider the solid phase of the bean to consist of just three main chemical reaction groups. We take these groups to be sucrose (with volume fraction $\phi_{1}$ ), reducing sugars (with volume fraction $\phi_{2}$ ), and other organic compounds (with volume fraction $\phi_{3}$ ). If we now recall that the solid volume fraction is denoted by $1-\phi$, we have that

$$
1-\phi=\sum_{i=1}^{3} \phi_{i}
$$

and hence the solid phase of the bean will have a volume fraction that varies in time and space as roating occurs.

We will consider reactions between the three groups using a simplified sugar pathway model. Firstly, sucrose hydrolyses into reducing sugars within the biological cells (at an effective reaction rate $\mathscr{R}_{3}(T)$ ). These reducing sugars then have two main reactions that they can undertake. Either they react to form $\mathrm{CO}_{2}$ gas and other organic solids via the Maillard reactions (with effective reaction rate $\mathscr{R}_{4}(T)$ and stoichiometric ratios of the products $\chi_{1}, \chi_{2}$ respectively), or they react to form $\mathrm{CO}_{2}$ and other organic solids via caramelization (with an effective reaction rate $\mathscr{R}_{5}(T)$ and stoichiometric ratios of the products $\chi_{3},\left(1-\chi_{1}-\chi_{2}-\chi_{3}\right)$ respectively).

The Maillard reaction (van Boekel, 2006) is a group of chemical reactions that describe the browning of various foodstuffs, from bread to malted barley to roasted coffee beans. These reactions involve amino acids combining with sugars to produce pyrazines, carbonyls, furrans, and other organic compounds (Martins et al., 2000). Contrastingly, the caramelization reactions are simply the pyrolysis of sugars and are thus viewed as entirely different reaction group. Nevertheless, the Maillard reaction relies on the presence of sugars to produce the aforementioned products of amino acids and therefore is included in this simplified sugar pathway.

Another important difference between the Maillard reactions and the caramelization reactions is that the Maillard reactions effectively act as an endothermic reaction group (Martins et al., 2000), whereas the caramelization reactions behave as an exothermic reaction group (Eggleston et al., 1996). Additionally, we assume that hydrolysis of sucrose can only occur if cell water is present. Using these simplified sugar pathway reactions, the conservation equations for the three solid phases are

$$
\begin{aligned}
\frac{\partial \phi_{1}}{\partial t} & =-\phi_{1}\left(\phi S-w_{b}-w_{n}\right) \mathscr{R}_{3}(T), \\
\frac{\partial \phi_{2}}{\partial t} & =\frac{\rho_{1}}{\rho_{2}} \phi_{1}\left(\phi S-w_{b}-w_{n}\right) \mathscr{R}_{3}(T)-\phi_{2}\left[\mathscr{R}_{4}(T)+\mathscr{R}_{5}(T)\right], \\
\frac{\partial \phi_{3}}{\partial t} & =\frac{\rho_{2}}{\rho_{3}} \phi_{2}\left[\chi_{2} \mathscr{R}_{4}(T)+\left(1-\chi_{1}-\chi_{2}-\chi_{3}\right) \mathscr{R}_{5}(T)\right] .
\end{aligned}
$$

Note that using (4.1), we can combine these three equations to give a conservation equation of the entire 


\begin{tabular}{cccc}
\hline Reaction Name and Number & $\begin{array}{c}\text { Global Reaction } \\
\text { Rate }\left(\alpha_{i}\right)\end{array}$ & $\begin{array}{c}\text { Exponential } \\
\text { Factor }\left(\beta_{i}\right)\end{array}$ & $\begin{array}{c}\text { Enthalpy, J } \\
\left(\lambda_{i}\right)\end{array}$ \\
\hline $\mathscr{R}_{1}(T)$ : Cell Degradation & 0.2 & 7.5 & $-1 \times 10^{5}$ \\
$\mathscr{R}_{2}(T)$ : Evaporation & 1 & 7 & $-2.3 \times 10^{6}$ \\
$\mathscr{R}_{3}(T):$ Sucrose Hydrolysis & 10 & 11.4 & $-4.3 \times 10^{4}$ \\
$\mathscr{R}_{4}(T)$ : Maillard Reactions & 0.01 & 10 & $-6.1 \times 10^{5}$ \\
$\mathscr{R}_{5}(T)$ : Caramelization & $1 \times 10^{-3}$ & 10 & $1 \times 10^{5}$ \\
\hline
\end{tabular}

Table 3. Parameters used in the global reaction rates $\mathscr{R}_{i}(T)$.

solid phase given by

$$
\begin{aligned}
\frac{\partial \phi}{\partial t}=( & \left.1-\frac{\rho_{1}}{\rho_{2}}\right) \phi_{1}\left(\phi S-w_{b}-w_{n}\right) \mathscr{R}_{3}(T) \\
& +\phi_{2}\left[\left(1-\frac{\rho_{2}}{\rho_{3}} \chi_{2}\right) \mathscr{R}_{4}(T)+\left(1-\frac{\rho_{2}}{\rho_{3}}\left(1-\chi_{1}-\chi_{2}-\chi_{3}\right)\right) \mathscr{R}_{5}(T)\right] .
\end{aligned}
$$

A summary of the parameter values used for three chemical reaction groups in the sugar pathway is shown in Table 3.

\subsection{Incorporating additional gas species}

Some of the chemical reactions in the Sugar Pathway Model also produce large quantities of $\mathrm{CO}_{2}$ gas. Hence, we need to not only to consider water vapour transport but also include the effects of $\mathrm{CO}_{2}$ in the model. We will also need to consider other gases; for example, when a bean is put into the roaster, it is typically full of normal atmospheric gases with a small amount of vapour and no $\mathrm{CO}_{2}$. Thus, we will lump all gases, other than water vapour and $\mathrm{CO}_{2}$, as a species that we call "air". We assume that the total gas pressure within a coffee bean can be represented as the sum of three partial gas pressures: air $\left(p_{a}\right), \mathrm{CO}_{2}\left(p_{c}\right)$, and water vapour $\left(p_{v}\right)$.

Transport of the gases in the bean has previously been assumed to be dominated by a bulk motion determined by a Darcy flow, driven by the total pressure gradient. The bulk motion is described through a permeability, which accounts for flow in nanopores that interconnect the pores in the structure. We note that, when fitted to data, the permeabilities required may be very small and perhaps non-physical. A second possible transport mechanism is to assume that the pores are completely sealed (a closed pore structure), so that transport is by adsorption into the structure and diffusion through the structure. A simple model of such a mechanism is an effective diffusion with flow driven by the partial pressure of the relevant gas. Since the mathematical form of these two mechanisms is very similar, we will adopt the notation of the Darcy flow but note that if permeabilities need to be very small, we might infer that the bean has a closed pore structure and diffusion dominates transport. We have not yet explored this diffusion mechanism in great detail.

We now consider the transport equations for the three gases. Firstly, the air does not participate in any reactions and hence the conservation equation of air implies

$$
\frac{\partial}{\partial t}\left[\frac{p_{a} \phi(1-S)}{T}\right]=\nabla \cdot\left[\frac{p_{a} k_{g}}{\mu T} \nabla\left(p_{a}+p_{c}+p_{v}\right)\right] .
$$


The $\mathrm{CO}_{2}$ gas is produced in both the caramelization and Maillard reactions and hence, conservation of this species requires that

$$
\frac{\partial}{\partial t}\left[\frac{p_{c} \phi(1-S)}{T}\right]=\nabla \cdot\left[\frac{p_{c} k_{g}}{\mu T} \nabla\left(p_{a}+p_{c}+p_{v}\right)\right]+\frac{R \rho_{2}}{m_{c}} \phi_{2}\left[\chi_{1} \mathscr{R}_{4}(T)+\chi_{3} \mathscr{R}_{5}(T)\right] .
$$

Finally, for the water vapour phase, we assume that all water lost from the liquid phase becomes water vapour, so that

$$
\frac{\partial}{\partial t}\left[\frac{p_{v} \phi(1-S)}{T}\right]=\nabla \cdot\left[\frac{p_{v} k_{g}}{\mu T} \nabla\left(p_{a}+p_{c}+p_{v}\right)\right]-\frac{R \rho_{w}}{m_{v}} \frac{\partial}{\partial t}[\phi S] .
$$

\subsection{Accounting for varying gas permeability}

We have assumed that the gases are driven through narrow pores and described using the permeability $k_{g}$. The pores in the structure are changing as the porosity varies and are constricted due to any water that is in them. Hence, the permeability needs to be modelled to account for these variations. The fundamental idea is that below a critical amount of gas, the gas phase sits in disconnected pockets and hence cannot move $\left(k_{g}=0\right)$ and that above this critical amount, the permeability increases as the amount of gas increases. Motivated by Zhang and Datta (2006), we assume that the permeability therefore depends only on the liquid volume fraction. A very simple model of this is

$$
k_{g}=k_{g 0} \max \left(1-\frac{\phi S}{\eta}, 0\right)
$$

where $k_{g 0}$ is the intrinsic gas permeability when there is no water present, and $\eta$ is a physical parameter between 0 and 1 that represents the threshold liquid fraction in the pore for gas to permeate. As this simple model originates from the study of moisture transport in bread baking, it seems reasonable that moisture transport in other organic materials (such as coffee beans) can also be described by this model.

\subsection{Effective thermal properties}

When considering the thermal energy in the bean, we must account for the heat released by the various chemical reaction groups that we have considered. In general, the heat released by each of the individual parts of any reaction group also follows a distribution across activation energies $E$. However, we will assume that the heat release is independent of $E$ and is constant (denoted by the positive constants $\lambda_{i}$ ). Note we assume that all reaction groups are net-endothermic (Martins et al., 2000) except the caramelization reaction group (Eggleston et al., 1996), which we assume to be net-exothermic (and account for this with a minus sign in the equations). Finally, as was done in Fadai et al. (2017), we will assume that at any point in space, all the different volumetric fractions are at the same temperature and that heat transport due to gas motion is negligible. Conservation of thermal energy can then be summarised by

$$
\begin{aligned}
\frac{\partial}{\partial t}\left[\rho_{\mathrm{eff}} C_{p, \mathrm{eff}} T\right]= & -\rho_{w}\left\{\lambda_{1} w_{b}\left[p_{v}-p_{v}^{*}(T, \phi S)\right] \mathscr{R}_{1}(T)+\lambda_{2}\left(\phi S-w_{b}-w_{n}\right) \mathscr{R}_{2}(T)\right\} \\
& -\lambda_{3} \rho_{1} \phi_{1}\left(\phi S-w_{b}-w_{n}\right) \mathscr{R}_{3}(T)-\rho_{2} \phi_{2}\left[\lambda_{4} \mathscr{R}_{4}(T)-\lambda_{5} \mathscr{R}_{5}(T)\right]+\nabla \cdot\left[K_{\mathrm{eff}} \nabla T\right]
\end{aligned}
$$

where $\rho_{\text {eff }}, C_{p, \text { eff }}$, and $K_{\text {eff }}$ are the effective thermal properties of the multiphase material. 
Because the gas phase stores very little heat, we will neglect the change in enthalpy provided by the gas phase, and define the effective thermal heat capacity by

$$
\rho_{\mathrm{eff}} C_{p, \mathrm{eff}}=\rho_{w} C_{p, w} \phi S+\sum_{i=1}^{3} \phi_{i} \rho_{i} C_{p, i}
$$

For the effective thermal conductivity in the multiphase bean, we use the simple form suggested in the literature for a randomly structured material (see, e.g., Wang and Pan (2008)) and determined by the harmonic average of the volume averaged thermal conductivities of the individual phases:

$$
K_{\mathrm{eff}}=\left(\frac{\phi S}{K_{w}}+\frac{\phi(1-S)}{K_{g}}+\sum_{i=1}^{3} \frac{\phi_{i}}{K_{i}}\right)^{-1},
$$

where $K_{g}$ is the thermal conductivity of the gas phase. This harmonic average was not used in previous models, such as Fadai et al. (2017), as the effects of a randomly structured material had not been considered. To determine $K_{g}$, one must consider the sum of the thermal conductivities of each gas, multiplied by their respective molar fraction. Noting that, because all the gases are locally at the same temperature, the molar fraction of each gas is identical to the fraction of each partial pressure, which yields

$$
K_{g}=\frac{K_{a} p_{a}+K_{c} p_{c}+K_{v} p_{v}}{p_{a}+p_{c}+p_{v}}
$$

This modelling is different from the approach taken in Fadai et al. (2017), where $K_{\text {eff }}$ was taken to be the arithmetic sum of the volume averaged thermal conductivities in each phase.

\subsection{The effective length scale of a whole coffee bean}

To better understand the length scale issues discussed in Section 2, we will consider a different geometry than that used by Fabbri et al. (2011) and Fadai et al. (2017) to represent a whole bean. This different geometry is motivated from the SEM image of the cross-section of a coffee bean shown in Figure 6. We conclude that a whole coffee bean can be quite well represented as a spherical "shell" of inner radius $a L$, where $0<a<1$, and outer radius $L$ as shown in Figure 6. We note that a half-spherical shell may be a more realistic representation of a coffee bean, but such details would break the spherical symmetry of the problem and hence preclude a simple one-dimensional analysis. For the bean chunks we can take a simple sphere of radius $r_{\text {chunk }}$ as the geometry.

We need to choose two constitutive equations in order to relate $a$ and $L$ to the effective radius of the bean $r_{e}$. Firstly, we assume that this spherical shell mapping should volume-preserving. Secondly, we impose that the average square-distance from any point in the bean to the boundary of the bean is preserved under the mapping. These two constitutive equations provide the framework for determining $a$ and $L$; in the context of a hemispherical coffee bean with effective radius $r_{e}$, this yields the equations

$$
\frac{2 \pi}{3} r_{e}^{3}=\frac{4 \pi}{3} L^{3}\left(1-a^{3}\right), \quad L(1-a)=\gamma r_{e},
$$

implying that

$$
a=\frac{1+\gamma^{3}-\sqrt{3 \gamma^{3}\left(2-\gamma^{3}\right)}}{1-2 \gamma^{3}}, \quad L=\frac{\gamma r_{e}}{(1-a)}
$$



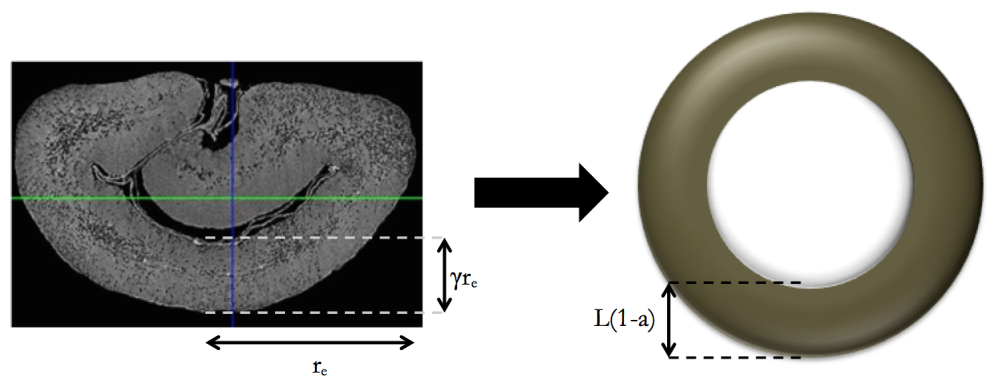

FIG. 6. SEM coffee bean, with measured radius $r_{e}$ and thickness $\gamma r_{e}$, and the idealised geometry of a spherical shell of outer radius $L$ and inner radius $a L$. The SEM image (left) is adapted from Fadai et al. (2017).

The constant $\gamma$ can be then determined directly from these or inferred from simple physical considerations: for example, using the fact that the drying time of coffee chunks in the experiment discussed in Section 2 takes roughly 65\% of the original whole bean drying time. Using this fact, and that the timescale of diffusive mechanisms scales with the square ratio of length scales, this gives us that

$$
\left(\frac{2 r_{\text {chunk }}}{L(1-a)}\right)^{2}=\left(\frac{2 r_{\text {chunk }}}{\gamma r_{e}}\right)^{2}=0.65
$$

By using the effective radii listed in Section 2, i.e. $r_{e}=4 \mathrm{~mm}$ and $r_{c h u n k}=0.7 \mathrm{~mm}$, this implies that

$$
\gamma=\frac{2 r_{\text {chunk }}}{r_{e} \sqrt{0.65}} \approx 0.43
$$

Indeed, with reference to Figure 6, it seems reasonable that the slab of bean material is approximately $43 \%$ of the effective radius of the whole bean, implying that $a \approx 0.47, L \approx 0.82 r_{e}$.

\subsection{Initial and boundary conditions}

Finally, we consider the initial and boundary conditions for the model. We assume, because the beans have been sitting in storage elsewhere for a long time, that they are filled with air with water vapour in equilibrium and no $\mathrm{CO}_{2}$ with a total pressure imposed by the surrounding atmosphere. For all geometries, we therefore impose uniform initial conditions on all variables with

$$
\begin{gathered}
\phi_{1}(r, 0)=\phi_{10}, \quad \phi_{2}(r, 0)=0, \quad \phi(r, 0)=\phi_{0}, \quad w_{b}(r, 0)=w_{b 0}, \quad S(r, 0)=\sigma, \\
T(r, 0)=T_{0}, \quad p_{a}(r, 0)=1 \mathrm{~atm}-p_{v}(r, 0)\left(=p_{a 0}\right), \quad p_{c}(r, 0)=0, \quad p_{v}(r, 0)=p_{v}^{*}\left(T_{0}, \phi_{0}, \sigma\right) .
\end{gathered}
$$

For the boundary conditions, we will need to consider what to do for the spherical shell (entire bean) and the sphere (chunks). We shall first make the assumption that any point on the surface of either of these geometries is easily accessible to the surrounding atmosphere. Therefore, after our spherical shell mapping, we will use the same boundary conditions on both the interior and exterior surface of the spherical shell. The surrounding atmosphere is controlled externally; here, we assume it has a total gas pressure of 1 atmosphere, has zero $\mathrm{CO}_{2}$ gas present, and a fixed vapour gas pressure. Hence, at any point on the boundary, we have that

$$
p_{a}+p_{c}+p_{v}=1 \mathrm{~atm}, \quad p_{c}=0, \quad p_{v}=p_{v 0} .
$$


In addition, motion of the surrounding atmosphere causes convection-dominated heat transfer at the surface of the bean (as discussed in Fadai et al. (2017)), and hence requires that

$$
K_{\mathrm{eff}} \nabla T \cdot \mathbf{n}=\left(\sum_{i} V_{f, i} h_{i}\right)\left(T_{\infty}-T\right),
$$

where $T_{\infty}$ is the controlled temperature of the roasting environment and $h_{i}$ are the convective coefficients of each phase. However, by making the assumption that $\left(\sum_{i} V_{f, i} h_{i}\right)$ can be represented by a bulk heat convective coefficient $h_{b}$, this heat transfer boundary condition can be simplified to

$$
K_{\text {eff }} \nabla T \cdot \mathbf{n}=h_{b}\left(T_{\infty}-T\right) .
$$

This gives us the boundary conditions we require for the system of equations at any surface.

Finally, if we are using the sphere geometry (chunks), we shall have to imposed regularity (i.e. symmetry) of the solution at the origin $(r=0)$.

\section{Non-dimensionlisation of the Sugar Pathway Model}

We nondimensionalise the model using the vapour diffusive timescale by letting

$$
t=\theta \hat{t}, \quad \text { where } \quad \theta=\frac{\phi_{0} \mu L^{2}}{k_{g 0} P_{0}}, \quad r=L \hat{r}, \quad P_{0}=1 \mathrm{~atm},
$$

and $L$ is the effective radius of the outer surface. We then set $\phi_{1}=\phi_{10} U_{1}, \phi_{2}=\phi_{10} U_{2}, \phi=\phi_{0} Q$, $w_{b}=w_{b 0} W, S=\sigma \hat{S}, T=T_{0}(1+\mathscr{T} \hat{T}), p_{a}=P_{0} P_{a}, p_{c}=P_{0} P_{c}, p_{v}=P_{0} P_{v}$, and $\mathscr{R}_{i}(T)=\alpha_{i} \hat{\mathscr{R}}_{i}(\hat{T})$, so that (after dropping the hat notation) our non-dimensional equations become

$$
\begin{aligned}
& \frac{\partial W}{\partial t}=\kappa_{1}\left(v Q S-W-v_{2}\right) \mathscr{R}_{1}(T)-\kappa_{2} W\left[P_{v}-P_{v}^{*}(T, Q, S)\right] \mathscr{R}_{2}(T), \\
& \frac{\partial}{\partial t}[Q S]=-\frac{\kappa_{2}}{v} W\left[P_{v}-P_{v}^{*}(T, Q, S)\right] \mathscr{R}_{2}(T), \\
& \frac{\partial U_{1}}{\partial t}=-\kappa_{3} U_{1}\left(v Q S-W-v_{2}\right) \mathscr{R}_{3}(T), \\
& \frac{\partial U_{2}}{\partial t}=\zeta_{1} \kappa_{3} U_{1}\left(v Q S-W-v_{2}\right) \mathscr{R}_{3}(T)-U_{2}\left[\kappa_{4} \mathscr{R}_{4}(T)+\kappa_{5} \mathscr{R}_{5}(T)\right], \\
& \frac{\partial Q}{\partial t}=\zeta_{2} \kappa_{3} U_{1}\left(v Q S-W-v_{2}\right) \mathscr{R}_{3}(T)+U_{2}\left[\zeta_{3} \kappa_{4} \mathscr{R}_{4}(T)+\zeta_{4} \kappa_{5} \mathscr{R}_{5}(T)\right], \\
& \frac{\partial}{\partial t}\left[\frac{P_{a} Q(1-\sigma S)}{1+\mathscr{T} T}\right]=\nabla \cdot\left[\frac{P_{a} k_{g}}{1+\mathscr{T} T} \nabla\left(P_{v}+P_{c}+P_{a}\right)\right], \\
& \frac{\partial}{\partial t}\left[\frac{P_{c} Q(1-\sigma S)}{1+\mathscr{T} T}\right]=\nabla \cdot\left[\frac{P_{c} k_{g}}{1+\mathscr{T} T} \nabla\left(P_{v}+P_{c}+P_{a}\right)\right]+U_{2}\left[\zeta_{5} \kappa_{4} \mathscr{R}_{4}(T)+\zeta_{6} \kappa_{5} \mathscr{R}_{5}(T)\right], \\
& \frac{\partial}{\partial t}\left[\frac{P_{v} Q(1-\sigma S)}{1+\mathscr{T} T}\right]=\nabla \cdot\left[\frac{P_{v} k_{g}}{1+\mathscr{T} T} \nabla\left(P_{v}+P_{c}+P_{a}\right)\right]-\zeta_{7} \frac{\partial}{\partial t}[Q S], \\
& \frac{\partial}{\partial t}[(1+\mathscr{T} T) H]=-\frac{\kappa_{1} \eta_{1}}{v}\left(v Q S-W-v_{2}\right) \mathscr{R}_{1}(T)-\frac{\kappa_{2} \eta_{2}}{v} W\left[P_{v}-P_{v}^{*}(T, Q, S)\right] \mathscr{R}_{2}(T) \\
& -\frac{\kappa_{3} \eta_{3}}{v} U_{1}\left(v Q S-W-v_{2}\right) \mathscr{R}_{3}(T)-\kappa_{4} \eta_{4} U_{2} \mathscr{R}_{4}(T) \\
& +\kappa_{5} \eta_{5} U_{2} \mathscr{R}_{5}(T)+\kappa_{6} \nabla \cdot\left[K_{\mathrm{eff}} \nabla T\right] .
\end{aligned}
$$


The boundary conditions at the external surface of the bean $(r=1)$ are then

$$
\frac{\partial T}{\partial r}=\frac{\mathrm{Nu}(1-T)}{K_{\mathrm{eff}}}, \quad P_{a}=\mathscr{P}_{1}, \quad P_{c}=0, \quad P_{v}=\mathscr{P}_{2} .
$$

For the spherical shell geometry, there are boundary conditions needed on the inner surface $(r=a)$ so that

$$
\frac{\partial T}{\partial r}=-\frac{\mathrm{Nu}(1-T)}{K_{\mathrm{eff}}}, \quad P_{a}=\mathscr{P}_{1}, \quad P_{c}=0, \quad P_{v}=\mathscr{P}_{2},
$$

while for the spherical geometry, regularity requires Neumann boundary conditions at the centre of the bean $(r=0)$ :

$$
\frac{\partial T}{\partial r}=\frac{\partial P_{a}}{\partial r}=\frac{\partial P_{c}}{\partial r}=\frac{\partial P_{v}}{\partial r}=0
$$

The initial conditions become

$$
\begin{array}{cccc}
U_{1}(r, 0)=1, & U_{2}(r, 0)=0, & Q(r, 0)=1, & W(r, 0)=1, \quad S(r, 0)=1, \\
T(r, 0)=0, & P_{a}(r, 0)=\mathscr{P}_{1}, & P_{c}(r, 0)=0, & P_{v}(r, 0)=\frac{D_{1} \exp \left(-D_{2}\right)}{1+C_{2}\left(\frac{1}{\phi_{0}}-1\right)} .
\end{array}
$$

In this problem, the nondimensional functions are defined as

$$
\begin{aligned}
\mathscr{R}_{i}(T)= & \exp \left(\beta_{i}(T-1)\right) \\
P_{v}^{*}(T, Q, S)= & \frac{D_{1}(Q S)^{C_{1}} \exp \left(\frac{D_{2}(T-1)}{1+\mathscr{T} T}\right)}{(Q S)^{C_{1}}+C_{2}\left(\frac{1}{\phi_{0}}-Q\right)^{C_{1}}}, \\
k_{g}= & \max (1-\tilde{v} Q S, 0) \\
H= & Q S+\omega_{1}\left(1-\phi_{0} Q\right)+\left(\omega_{2}-\phi_{10} \omega_{1}\right) U_{1}+\left(\omega_{3}-\phi_{10} \omega_{1}\right) U_{2}, \\
K_{\mathrm{eff}}= & {\left[Q S+\omega_{4}\left(1-\phi_{0} Q\right)+\left(\omega_{5}-\phi_{10} \omega_{4}\right) U_{1}+\left(\omega_{6}-\phi_{10} \omega_{4}\right) U_{2}+\frac{\omega_{7} Q(1-\sigma S)}{K_{g}}\right]^{-1}, } \\
K_{g}= & \frac{P_{v}+\omega_{8} P_{c}+\omega_{9} P_{a}}{P_{v}+P_{c}+P_{a}},
\end{aligned}
$$

and all the nondimensional parameters that have been introduced are defined in Table 4 with typical values shown where known.

\section{Numerical simulations and comparison with experiments}

We solve the Sugar Pathway Model using the method of lines exploiting a second-order accurate finite difference scheme in $r$. Using the stiff ODE solver ode15s in MATLAB, we can obtain the solution to the Sugar Pathway Model described in Section 5. In order to compare these predictions to experimental data, we can use the solutions to compute the average moisture content in a bean. For the spherical shell, this average moisture content is given by

$$
M_{a v g}(t)=\frac{3 \rho_{w} \phi_{0} \sigma}{\rho_{b}\left(1-a^{3}\right)} \int_{a}^{1} Q(r, t) S(r, t) r^{2} d r
$$




\begin{tabular}{|c|c|c|c|}
\hline $\begin{array}{l}\text { Dimensionless } \\
\text { Grouping }\end{array}$ & $\begin{array}{c}\text { Relationship to } \\
\text { Dimensional Parameters }\end{array}$ & $\begin{array}{l}\text { Typical value of } \\
\text { Parameter }\end{array}$ & Reference \\
\hline$C_{1}$ & - & 0.41 & Corrêa et al. (2010) \\
\hline$C_{2}$ & - & $6.1 \times 10^{-3}$ & Corrêa et al. (2010) \\
\hline$D_{1}$ & - & 35.0 & Dean (1999) \\
\hline$D_{2}$ & - & 7.31 & Dean (1999) \\
\hline$v$ & $\frac{\phi_{0} \sigma}{w_{b 0}}$ & 100 & Experimentally determined \\
\hline$v_{2}$ & $\begin{array}{l}w_{b 0} \\
\frac{w_{n}}{w_{b 0}}\end{array}$ & 10 & Chosen to fit data \\
\hline$\tilde{v}$ & $\frac{\phi_{0} \sigma}{n}$ & 0.1 & Chosen to fit data \\
\hline$\kappa_{1}$ & $\alpha_{1} \theta$ & $2.3 \times 10^{-4}-5.1 \times 10^{-3}$ & Chosen to fit data \\
\hline$\kappa_{2}$ & $P_{0} \alpha_{2} \theta$ & $120-2600$ & Chosen to fit data \\
\hline$\kappa_{3}$ & $w_{b 0} \alpha_{3} \theta$ & $4.7 \times 10^{-6}-1.0 \times 10^{-5}$ & Chosen to fit data \\
\hline$\kappa_{4}$ & $\alpha_{4} \theta$ & $1.2 \times 10^{-5}-2.6 \times 10^{-4}$ & Chosen to fit data \\
\hline$\kappa_{5}$ & $\alpha_{5} \theta$ & $1.2 \times 10^{-6}-2.6 \times 10^{-5}$ & Chosen to fit data \\
\hline$\kappa_{6}$ & $\frac{K_{w}}{\rho_{w} C_{p w}} \cdot \frac{\mu}{k_{o 0} P_{0}} \cdot \frac{\mathscr{T}}{\phi_{0} \sigma^{2}}$ & 0.14 & Krupińska et al. (2007) \\
\hline$\zeta_{1}$ & 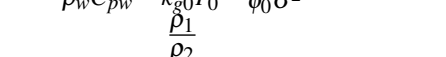 & 0.97 & Ginz et al. (2000) \\
\hline$\zeta_{2}$ & $\left(1-\frac{\rho_{1}}{\rho_{2}}\right) \frac{\phi_{10}}{\phi_{0}}$ & $3.0 \times 10^{-3}$ & Ginz et al. (2000) \\
\hline$\zeta_{3}$ & $\left(1-\frac{\rho_{2} \chi_{2}}{\rho_{3}}\right) \frac{\phi_{10}}{\phi_{0}}$ & 0.067 & Ginz et al. (2000) \\
\hline$\zeta_{4}$ & $\left(1-\frac{\rho_{2}}{\rho_{3}}\left(1-\chi_{1}-\chi_{2}-\chi_{3}\right)\right) \frac{\phi_{10}}{\phi_{0}}$ & 0.067 & Ginz et al. (2000) \\
\hline$\zeta_{5}$ & $\frac{R T_{0} \rho_{2} \phi_{10} \chi_{1}}{P_{0} m_{c} \phi_{0}}$ & 27 & Ginz et al. (2000) \\
\hline$\zeta_{6}$ & $\frac{R T_{0} \rho_{2} \phi_{10} \phi_{0} \chi_{3}}{P_{0} m_{0} \phi_{0}}$ & 9.0 & Ginz et al. (2000) \\
\hline$\zeta_{7}$ & $\frac{R T_{0} \rho_{w} \sigma}{P_{0} m_{v}}$ & 110 & Ginz et al. (2000) \\
\hline$\omega_{1}$ & $\frac{\rho_{3} C_{p 3}}{\rho_{w} C_{p w} \phi_{0} \sigma}$ & 0.062 & Chosen to fit data \\
\hline$\omega_{2}$ & $\frac{\rho_{1} C_{p 1} \phi_{10}}{\rho_{w} C_{p w} \phi_{0} \sigma}$ & 1.8 & Ginz et al. (2000) \\
\hline$\omega_{3}$ & $\frac{\rho_{2} C_{p 2} \phi_{10}}{\rho_{w} C_{p w} \phi_{0} \sigma}$ & 0.58 & Chosen to fit data \\
\hline$\omega_{4}$ & $\frac{K_{w}}{K_{3} \phi_{0} \sigma}$ & 240 & Ginz et al. (2000) \\
\hline$\omega_{5}$ & $\frac{K_{w} \phi_{0}}{K_{1} \phi_{0} \sigma}$ & 1.4 & Ginz et al. (2000) \\
\hline$\omega_{6}$ & $\frac{K_{w} \phi_{10}}{K_{2} \phi_{0} \sigma}$ & 2.4 & Ginz et al. (2000) \\
\hline$\omega_{7}$ & $\frac{K_{w}}{K_{y} \sigma}$ & 450 & Experimentally determined \\
\hline$\omega_{8}$ & $\frac{K_{a}}{K_{y}}$ & 1.5 & Fadai et al. (2017) \\
\hline$\omega_{9}$ & $\frac{\hat{K}_{c}^{y}}{K_{v}}$ & 0.94 & Fadai et al. (2017) \\
\hline$\eta_{1}$ & $\frac{\lambda_{1}^{v}}{T_{0} C_{p w}}$ & 2.4 & Chosen to fit data \\
\hline$\eta_{2}$ & $\frac{\lambda_{2}}{T_{0} C_{p w}}$ & 1.9 & Fadai et al. (2017) \\
\hline$\eta_{3}$ & $\frac{\rho_{1} \lambda_{3} \phi_{10}}{\rho_{w} T_{0} C_{p w} \phi_{0} \sigma}$ & $2.8 \times 10^{-3}$ & Chosen to fit data \\
\hline$\eta_{4}$ & $\frac{\rho_{2} \phi_{10} \lambda_{4}}{\rho_{w} \phi_{0} \sigma T_{0} C_{p w}}$ & 0.41 & Chosen to fit data \\
\hline$\eta_{5}$ & $\frac{\rho_{2} \phi_{10} \lambda_{5}}{\rho_{w} \phi_{0} \sigma T_{0} C_{p w}}$ & $6.7 \times 10^{-3}$ & Chosen to fit data \\
\hline $\mathscr{P}_{1}$ & $\frac{p_{a 0}}{P_{0}}$ & 0.98 & Experimentally determined \\
\hline $\mathscr{P}_{2}$ & $\frac{p_{v 0}}{P_{0}}$ & 0.024 & Experimentally determined \\
\hline $\mathrm{Nu}$ & $\frac{h_{b} L \phi_{0} \sigma}{K_{w}}$ & $1.5-6.9 \times 10^{-4}$ & Chosen to fit data \\
\hline $\mathscr{T}$ & $\frac{T_{\infty}{ }^{N} w}{T_{0}}-1$ & 0.72 & Experimentally determined \\
\hline
\end{tabular}

Table 4. Description and typical values of dimensionless groupings used in the Sugar Pathway Model. The range of values for $\kappa_{i}$ and $\mathrm{Nu}$ are based on using lengths from the chopped bean radius $(L=0.7 \mathrm{~mm})$ to the whole bean spherical shell $(L=3.3 \mathrm{~mm})$. 
where $\rho_{b}$ denotes the bulk density of the coffee bean, and the same formula is valid for the solid sphere geometry by setting $a=0$. For the calculations presented here, the chopped bean used a value of $L=0.7 \mathrm{~mm}$ while the whole bean used $a=0.47, L=3.3 \mathrm{~mm}$.

To fit the data, we have informally chosen parameter values to get a good fit to the experimental data for moisture content. However, we appear to be able to choose values for the permeability over a vast range but we can still get a good fit by appropriately choosing $h_{b}=3.0-3.5 \mathrm{Wm}^{-2} \mathrm{~K}^{-1}$, the heat transfer coefficient. To illustrate this, we have chosen to fit the data with two different values of permeability. The first is that appropriate to Darcy flow in timber of $2.5 \times 10^{-14} \mathrm{~m}^{2}$, which is similar to values of wood permeabilities seen in Krupińska et al. (2007). The second comes from estimates made on a different experiment using coffee beans (Anderson et al., 2003). After coffee beans have been roasted, it is very common to quickly quench them to room temperature and then allow them to temper. This tempering is done by placing the beans in a bag and leaving them for several hours. During this rest period, significant quantities of gas come out of the bean and can be seen to inflate the bag. In Anderson et al. (2003), a very simple approach is taken to how this gas behaves and an estimate is made of the diffusion coefficient necessary to see the length decay rate. There has been no other substantive modelling or experimental data collected on this behaviour, so this value is speculative but given as $2.5 \times 10^{-16} \mathrm{~m}^{2}$. We will use this value as our second value for fitting the Sugar Pathway Model.

The predictions of the model using these two values of permeability, with $h_{b}$ chosen to make the fit, are shown in Figure 7, and show the Sugar Pathway Model has good agreement with the two experimental data sets of the chopped bean experiment. It is important to note that the last two data points of the roasted whole bean experiment are after an event known as first crack (Schenker, 2000), where macroscopic deformation of the coffee bean has occurred. In consequence, these two data points may be affected by mechanisms not included in the Sugar Pathway Model. This demonstrates that the issues concerning length scale have now been resolved and that there is a range of possible permeabilities that might be reasonable.

In both parameter cases, as was seen in Fadai et al. (2017), the timescale for thermal diffusion across the bean is much smaller than the timescale for gas flow (i.e. $\kappa_{6} \ll 1$ ). This implies that the temperature in the bean is nearly spatially uniform throughout the entire roasting process (see Figure 8(a)). This then implies that the global chemical reaction rates $\mathscr{R}_{i}(T)$ will also be nearly spatially uniform and cause $Q, U_{1}, U_{2}$ and $S$ to display similar features. One notable exception to this, however, is in the early roasting time for $W$. With reference to Figure 8(b), we see that the bound water content is driven to very small levels early in the roasting and displays similar features to the drying front discussed in Fadai et al. (2018). However, note that in these regions of small bound water (when $W \ll 1$ ), the cell water that is released at a rate determined by $\mathscr{R}_{1}(T)$ immediately evaporates. Thus, the mechanism that slows the overall evaporation rate is linked to the degradation rate $\mathscr{R}_{1}(T)$ rather than the gas permeability $k_{g}$.

For the large permeability case, we note that the gas species are nearly spatially uniform, as seen in Figure 9(a). This is due to the fact that the removal of water vapour from the bean is controlled by the degradation reaction, described by $\mathscr{R}_{1}(T)$, and the uniform temperature is being restricted by the low heat transfer coefficient. Once the cell degrades, the water vapour is easily transported to the surface by Darcy flow. For the very small permeability case, as seen in Figure 9(b), the gas pressures vary significantly and there is a drying front that travels through the bean. In this case, the cell degradation rate is relatively rapid, so that the water can evaporate quickly but is then severely restricted in diffusing out of the bean, creating large gas pressures. Note that in this case, the chemical reactions are not as spatially uniform, as the hydrolysis reaction $\mathscr{R}_{3}(T)$ depends on water and the presence of the drying front makes this occur at different rates in different parts of the bean. 


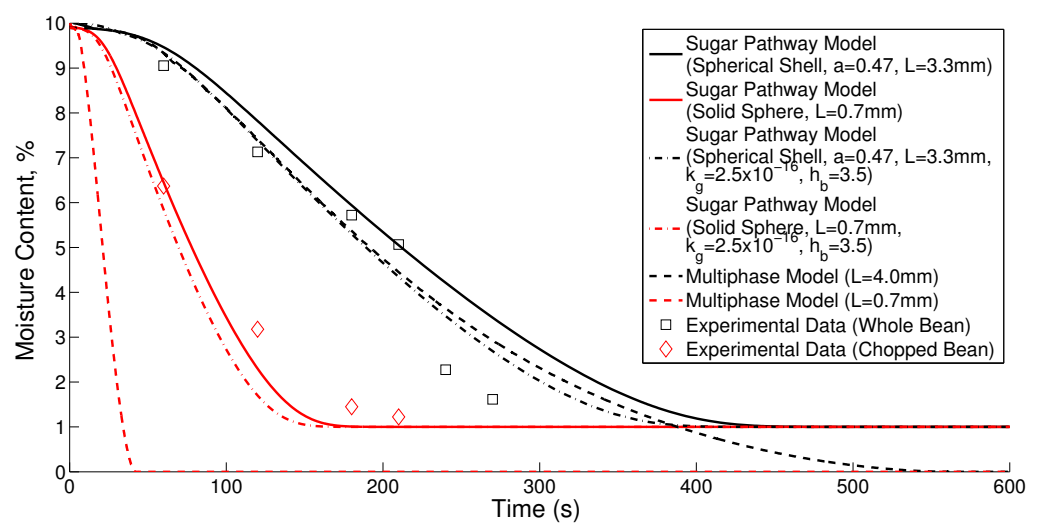

FIG. 7. Comparison of the average moisture content in a bean chunk versus a whole bean during a $230^{\circ} \mathrm{C}$ roast. The solid and dot-dashed lines correspond to the average moisture loss determined by the Sugar Pathway Model described in Section 5, with parameters (unless otherwise listed in the legend) obtained from Table 4. The dashed lines correspond to the average moisture loss determined by the Multiphase Model presented in Fadai et al. (2017), and the markers correspond to experimental data seen in Table $1 ; h_{b}=3.0$ unless otherwise stated.

\subsection{Parameter Sensitivity}

The Sugar Pathway Model has 19 dimensionless groups that have unknown values. Hence, it is appropriate to consider how sensitive the Sugar Pathway Model is to changing values of dimensionless parameter values. With reference to Figure 10, we see four general qualitative features that occur when altering values of these dimensionless groups. We focus on the sensitivity of the temperature and moisture content of the bean due to the lack of experimental data for the other variables.

The first qualitative feature seen is a "faster reaction" effect, where an increase (or decrease) in a parameter's value causes the bean to heat up on a shorter timescale. This can also be seen in the bean's average moisture content, where a different parameter value causes the moisture to be lost at a faster rate. We observe this phenomena when $\omega_{1}, \omega_{3}, \kappa_{2}, \eta_{1}$, and $\eta_{4}$ are decreased, or $h_{b}$ is increased, from the parameter values stated in Table 4. As previously mentioned in this section, we can allow for a smaller gas permeability value $k_{g}$ if we increase $h_{b}$. Since these other dimensionless groupings exhibit similar qualitative changes when their parameter values are decreased, we infer that one could use each parameter value to compensate for a smaller value of $k_{g}$ and still match the experimental data.

Another feature seen in certain parameter regimes is a stronger effect of the exothermic caramelisation reaction group. Naturally, this is observed when $\eta_{5}$ is increased, as this corresponds to more energy being produced from the caramelisation reaction group and therefore causes the bean to heat up faster, even surpassing the roasting temperature in some cases. Curiously, however, we also see similar phenomena when $\omega_{3}$ or $\kappa_{3}$ increases, as well as when $\beta_{3}$ decreases. We infer that when the reducing sugars are easier to react via the caramelisation reaction group (such as in the case of larger $\kappa_{3}$ and smaller $\beta_{3}$ ) and more energy is released into the system.

We find that the bean's temperature changes for intermediate time when $\kappa_{1}$ and $\beta_{1}$ are changed. 


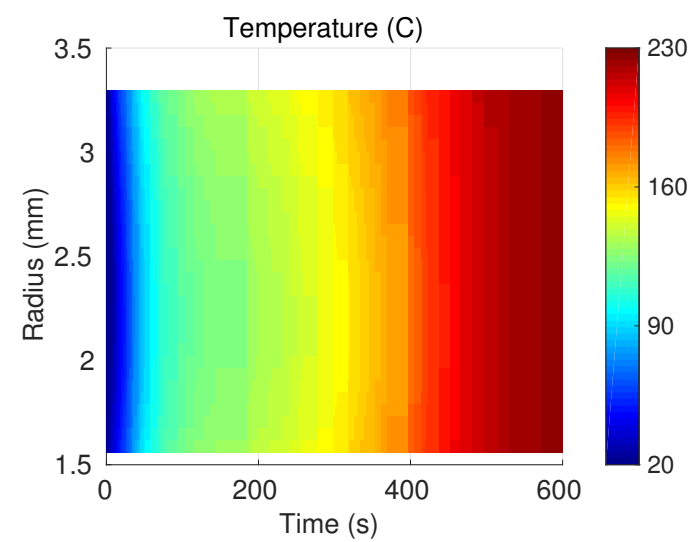

(a)

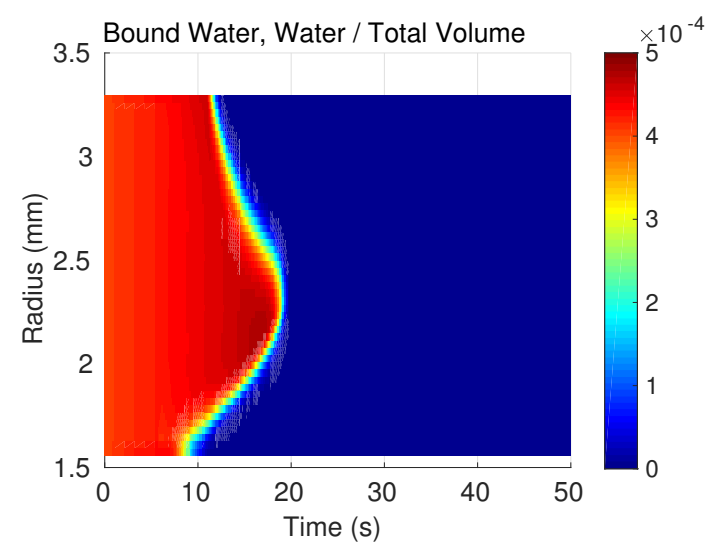

(b)

FIG. 8. (a) Temperature $T(r, t)$ over a 600 -second roast at $230^{\circ} \mathrm{C}$ in the spherical shell geometry. (b) The short-time behaviour for $W(r, t)$ in the spherical shell geometry. All parameters for both figures are the upper bound of values listed in Table 4 .

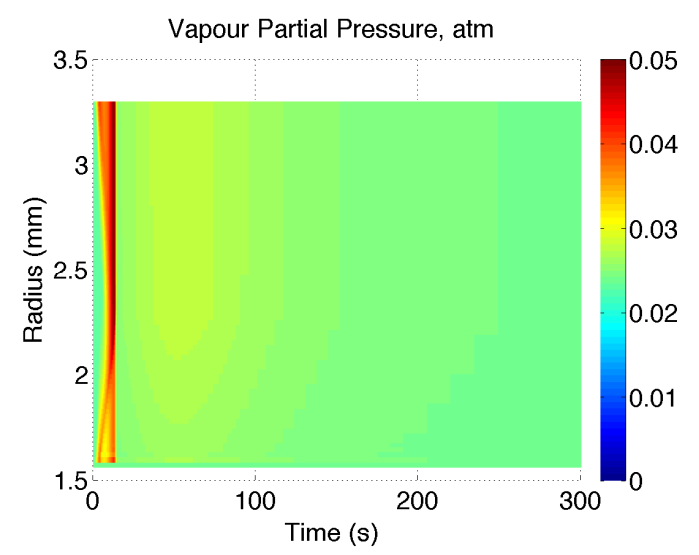

(a)

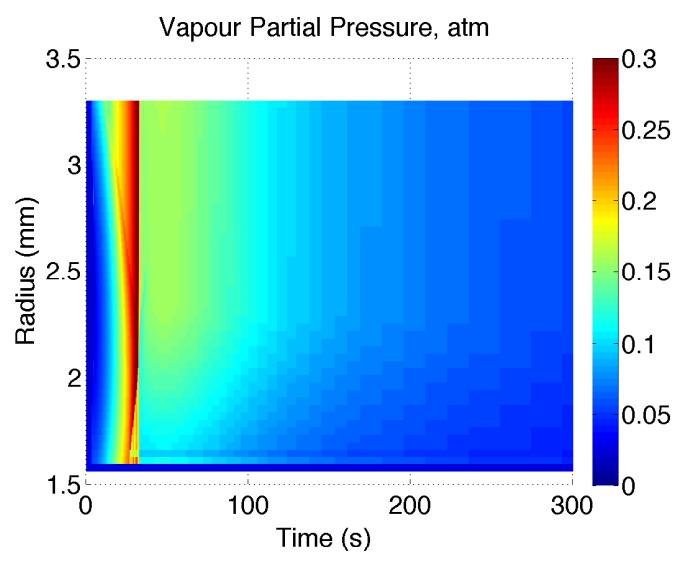

(b)

FIG. 9. The partial vapour pressure $p_{v}(r, t)$ for the first 300 seconds of a $230^{\circ} \mathrm{C}$ roast in the spherical shell geometry, with (a) $k_{g}=2.5 \times 10^{-14}$ and $h_{b}=3$; (b) $k_{g}=2.5 \times 10^{-16}$ and $h_{b}=3.5$. All other parameters for both figures are the upper bound of values listed in Table 4. 
Faster Reactions (R)
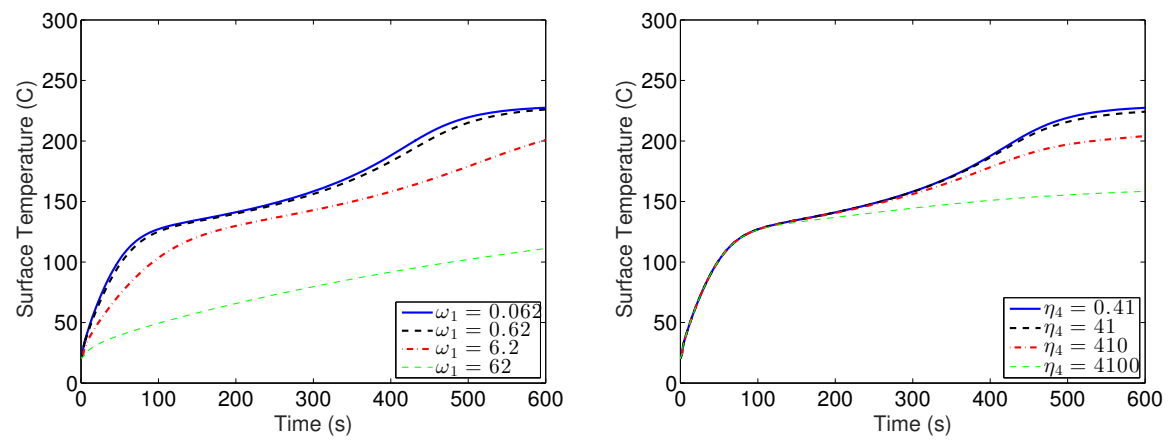

Larger Exothermic Influence (E)
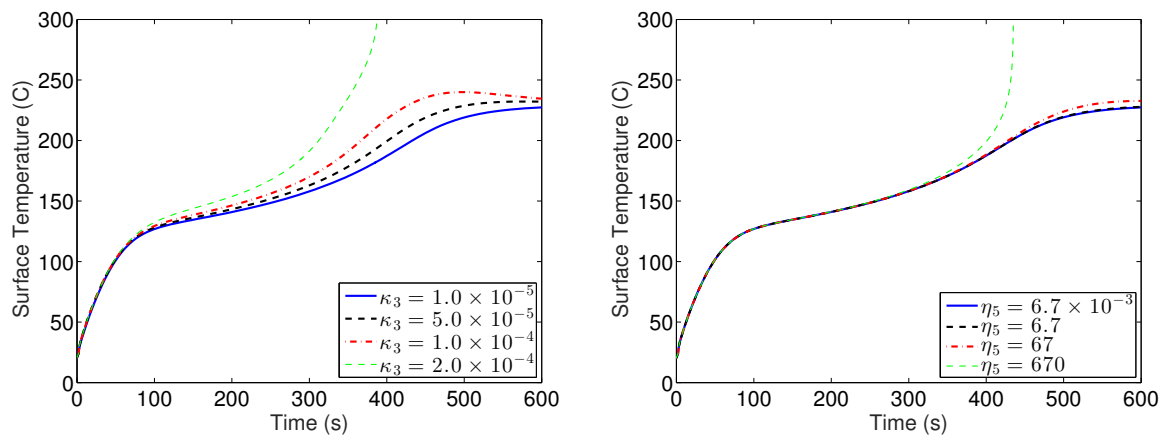

Qualitative Changes in Temperature (QT)
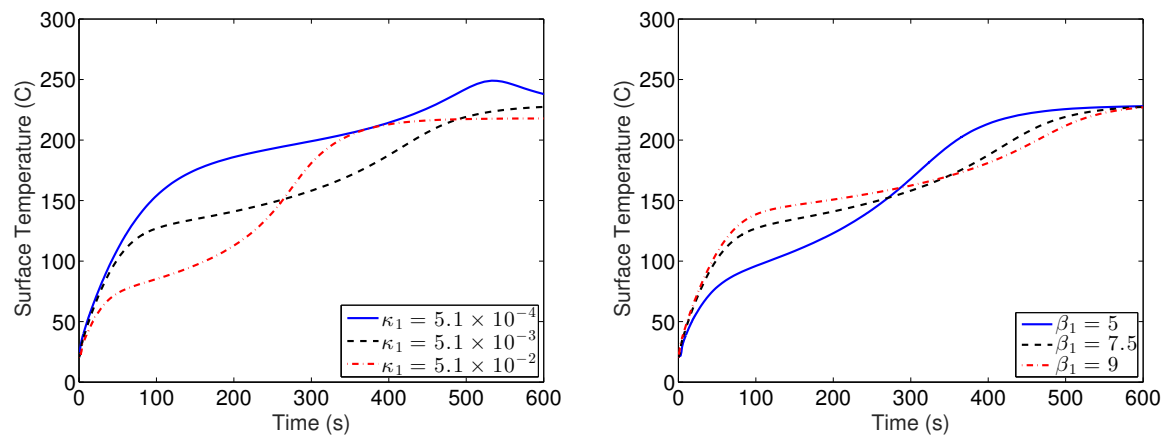

Qualitative Changes in Moisture/Vapour (QM)
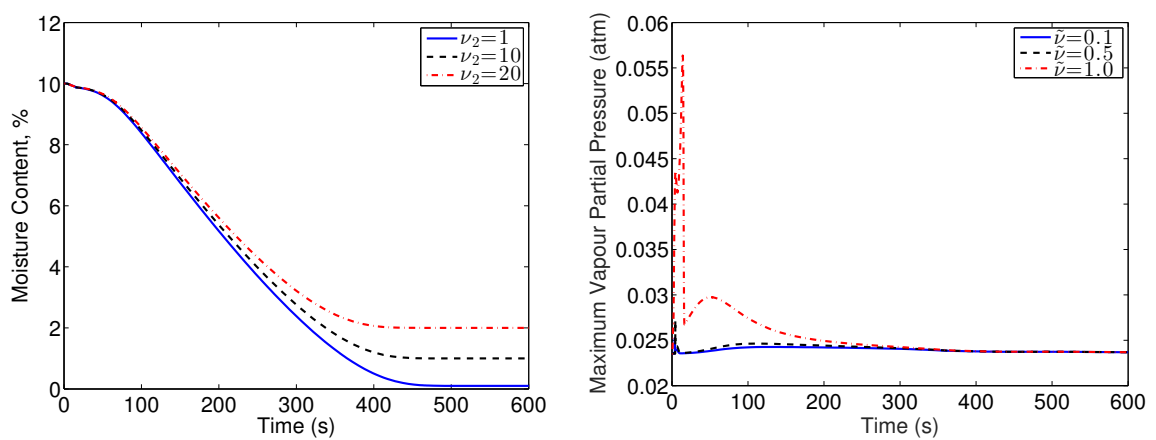

FIG. 10. Qualitative changes observed for various values of dimensionless groupings. All parameters for both figures are the upper bound of values listed in Table 4 unless specified in the figure. 


\begin{tabular}{cc}
\hline Qualitative Feature & Dimensionless Grouping \\
\hline $\mathrm{R}$ & $\operatorname{inc} h_{b} ; \operatorname{dec} \omega_{1}, \omega_{3}, \kappa_{2}, \eta_{1}, \eta_{4}$ \\
$\mathrm{E}$ & $\operatorname{inc} \omega_{3}, \kappa_{3}, \eta_{5} ; \operatorname{dec} \beta_{3}$ \\
QT & $\kappa_{1}, \beta_{1}$ \\
QM & $v_{2}, \tilde{v}(\operatorname{provided} \tilde{v} \leqslant 1)$ \\
(No significant change observed) & $\kappa_{4}, \kappa_{5}, \beta_{2}, \beta_{4}, \beta_{5}, \eta_{3}$ \\
\hline
\end{tabular}

Table 5. A summary of the sensitivity of dimensionless groupings used in the Sugar Pathway Model by qualitative features. Faster reactions (R) and a larger exothermic influence (E) are observed when certain parameters are increased (inc) or decreased (dec), while qualitative changes in temperature (QT) and moisture/vapour content (QM) are observed in others. See Figure 10 for the beahviour of each feature.

This is to be expected, as the cell degradation reaction group determines the free water content in the bean, which is then used to hydrolyse sucrose in the bean. If this reaction is slower than later reactions (e.g. larger $\beta_{1}$ or smaller $\kappa_{1}$ ), then hydrolysed sucrose will immediately be consumed by the Maillard and caramelisation reaction groups. Therefore, an understanding of the parameters used in the cell degradation reaction group is critical in determining the various products in the Sugar Pathway Model: in particular, the Maillard and caramelisation products.

We see changes in the final moisture content when $v_{2}$ is varied. This is to be expected, since a larger value of $v_{2}$ implies having a larger quantity of inaccessible water that will remain for the duration of the roast. Indeed, the value of $v_{2}$ corresponds to the percentage of initial water remaining as $t \rightarrow \infty$ (so, $v_{2}=$ 10 implies that $10 \%$ of the initial water content in the bean will remain after roasting). This parameter can therefore be determined via further "chopped green coffee bean experiments" to determine how much inaccessible water is present in a coffee bean. Additionally, we see a larger vapour pressure only for larger values of $\tilde{v}$. When $\tilde{v}>1$, however, the vapour transport mechanism described in Section 4.3 in invalid and a different model for variable gas permeability should be used. However, typical values of $\tilde{v}$ indicate that $\tilde{v} \approx 0.1$ (Zhang and Datta, 2006), so we anticipate that our variable permeability model is reasonably valid.

Finally, there are a large number of dimensionless groupings that yield no significant changes to the predictions of the Sugar Pathway Model. Specifically, no changes in the qualitative features of temperature and moisture content were observed for various values of $\kappa_{4}, \kappa_{5}, \beta_{2}, \beta_{4}, \beta_{5}$, and $\eta_{3}$. A summary of all the different qualitative features is shown in Table 5.

\section{Discussion}

In this paper, we have incorporated modified evaporation rates and chemical reaction groups to improve existing mathematical models of roasting coffee beans. These additions were motivated both by the inability of previous mathematical models (see Fabbri et al. (2011); Fadai et al. (2017, 2018)) to reproduce experimental data at different length scales as well as some physically unrealistic parameter values. These limitations were discussed and we provided a brief overview of the existing models presented in Fabbri et al. (2011), Fadai et al. (2017), and Fadai et al. (2018). In particular, the chopped green coffee bean thought experiment discussed in Fadai et al. (2017) was undertaken and data compared with predictions. The experiments suggested that the evaporation mechanism presented in Fadai et al. (2017) did not reflect moisture transport in a roasting bean with sufficient accuracy, and hence a different approach was required to model the evaporation rate inside a roasting bean.

We presented a model of evaporation of the water in a roasting coffee bean in two stages, the first 
being release of cell water into bound water by degradation of the cell and the second by bound water evaporating. We considered evaporation of bound water using the ideas of chemical reaction groups. By assuming that this chemical reaction group has a distribution of activation energies the evaporation rate had a global reaction rate. We also incorporated how evaporation is affected by deviation of vapour pressure from an equilibrium vapour pressure of the bound water. This equilibrium vapour pressure has to account for the large number of possible binding sites in the material and was described using the sorption isotherm of the material.

Motivated by the global reaction rate we then used these ideas to account for additional chemical reactions, relevant to flavour development. As these reactions are complicated, we examined a simplified sugar pathway network, which turns sucrose into $\mathrm{CO}_{2}$ gas and other solids via reducing sugars. Modelling this pathway also required us to model the transport of gases other than water vapour (in this case, $\mathrm{CO}_{2}$ and air) within the bean, as well as the energy produced or consumed during these additional chemical reactions. We also revisited the idealised geometry to use for a coffee bean. We considered a spherically symmetric shell geometry to be a more appropriate representation of a whole coffee bean than a solid sphere. We also considered that chunks of bean, as used in the chopped bean experiment, are adequately represented by solid spheres.

We solved the Sugar Pathway Model numerically, and were able to compare our simulations to data obtained from roasting experiments we performed on both solid coffee beans and chopped green coffee beans. Indeed, we found that with a more suitable choice of geometries, the sugar pathway model agrees well with the moisture data obtained from experiments. However, we also found that we needed to choose combinations of the heat transfer coefficient and the permeability in order to make a good fit, but that we could not identify what combination was appropriate just by fitting the moisture content data. This lack of parameter identifiability is due to the fact that the rate at which moisture is lost can be controlled in the model either by altering the temperature, so that the cell degradation controls evaporation, or altering the permeability, to restrict the rate at which the water vapour is transported to the surface. Any combination of these two effects that give the same overall restriction to water vapour transport will fit the moisture data.

We undertook a sensitivity analysis to examine how model solutions depend on parameters whose values were not known from independent sources. We found four main types of qualitative behaviours, namely: faster reactions, stronger dependence on exothermic reaction groups, different thermal behaviours for intermediate time, and different final moisture contents. We also determined the relevant nondimensional parameters controlling each of these behaviours. While one could improve the fit of the model with additional experimental data, the physically relevant behaviour has been identified via a few notable dimensionless groups. Hence, we believe that the general qualitative behaviour we have identified is robust to the parameters in the Sugar Pathway Model.

In order to gain an even better understanding of the Sugar Pathway Model we proposed here, certain questions might be addressed in the future. First, changes in the permeability create significant alterations in the predicted size of the internal gas pressure within the coffee bean. Finding experimental data that could identify what these pressures are is key to understanding the behaviour that occurs and in identifying possible spacial alterations in the chemical reactions that occur. We see three possible candidate for such sources of data. The first is detailed measurements the flow of gas during tempering of beans after roasting. The second is to directly measure the sorption isotherm of roasted beans. The third is to examine more careful the changes that occur to a bean at "first crack", which is a phenomena very similar to the popping of popcorn and may rely on high pressure to cause mechanical deformation of the bean. Given that the model has the ability to allow large variations in the parameters and still explain the moisture content data, we anticipate that new data sources will then give significant insight 
into the behaviour within the beans.

We note that several non-dimensional parameters can be seen to be of very different orders of magnitude, and hence their size could be exploited to perform asymptotic analysis on the model. This would be beneficial for general understanding of the underlying evaporation and chemical reactions in the model. Finally, the models could be further extended to account for volumetric expansion and deformation of the coffee bean that might be key to understanding the mechanisms underlying of "first crack". Such incorporations would allow one to represent the coffee bean solid matrix through the roasting process in a more realistic fashion.

\section{Acknowledgments}

This publication is based on work supported by the EPSRC Centre for Doctoral Training in Industrially Focused Mathematical Modelling (EP/L015803/1) in collaboration with Jacobs Douwe Egberts. N. Fadai thanks Jacobs Douwe Egberts for financial support and the opportunity to work on-site during parts of this project.

\section{REFERENCES}

B. A. Anderson, E. Shimoni, R. Liardon, T. P. Labuza, The diffusion kinetics of carbon dioxide in fresh roasted and ground coffee, Journal of Food Engineering 59 (2003), 71-78.

J. Baggenstoss, Coffee roasting and quenching technology - Formation and stability of aroma compounds, $\mathrm{PhD}$ thesis (2008), Swiss Federal Institute of Technology.

P. C. Corrêa, A. L. D. Goneli, P. C. A. Júnior, G. H. H. de Oliveira, D. S. M. Valente, Moisture sorption isotherms and isosteric heat of sorption of coffee in different processing levels, International Journal of Food Science and Technology 45 (2010), 2016-2022.

G. L. Comstock, Directional permeability of softwoods, Wood and Fiber Science 4 (1970), 283-289.

J. A. Dean, Lange's Handbook of Chemistry, 15th Ed. (1999), McGraw-Hill, New York.

G. Eggleston, B. J. Trask-Morrell, J. R. Vercellotti, Use of differential scanning calorimetry and thermogravimetric analysis to characterize the thermal degradation of crystalline sucrose and dried sucrose-salt residues, Journal of Agricultural and Food Chemistry 44 (1996), 3319-3325.

A. Fabbri, C. Cevoli, L. Alessandrini, and S. Romani, Numerical modeling of heat and mass transfer during coffee roasting process, Journal of Food Engineering, 105 (2011), 264-269.

N. T. Fadai, J. Melrose, C. P. Please, A. Schulman, R. A. Van Gorder, A Heat and Mass Transfer Study of Coffee Bean Roasting, International Journal of Heat and Mass Transfer 104 (2017), 787-799.

N. T. Fadai, C. P. Please, R. A. Van Gorder, Asymptotic Analysis of a Multiphase Drying Model Motivated by Coffee Bean Roasting, SIAM Journal on Applied Mathematics 78 (2018), 418-436.

M. Ginz, H. H. Balzer, A. G. W. Bradbury, H. G. Maier, Formation of aliphatic acids by carbohydrate degradation during roasting of coffee, European Food Research and Technology 211 (2000), 404-410.

B. Krupińska, I. Strømmen, Z. Pakowski, T. M. Eikevik, Modelling of sorption isotherms of various kinds of wood at different temperature conditions, Drying Technology 25 (2007), 1463-1470.

K. J. Laidler, The development of the Arrhenius equation, Journal on Chemical Education 61 (1984), 494-498.

I. Langmuir, The Vapour Pressure of Metallic Tungsten, Physical Review 2 (1919), 329-342.

S. I. Martins, W. M. Jongen, M. A. Van Boekel, A review of Maillard reaction in food and implications to kinetic modelling, Trends in Food Science and Technology 11 (2000), 364-373.

C. R. Oswin, The kinetics of package life: III. Isotherm, Journal of the Society of Chemical Industry 65 (1946), 419-421.

P. Perré and I. W. Turner, A 3-D version of TransPore: a comprehensive heat and mass transfer computational model for simulating the drying of porous media, International Journal of Heat and Mass Transfer 42 (1999), 
4501-4521.

C. P. Please, M. J. McGuinness, D. L. S. McElwain, Approximations to the distributed activation energy model for the pyrolysis of coal, Combustion and Flame 133 (2003), 107-117.

$\mathrm{S}$. Schenker, Investigations on the hot air roasting of coffee, $\mathrm{PhD}$ thesis (2000), Swiss Federal Institute of Technology, Zurich.

J. M. Talbot, Grounds for agreement: The political economy of the coffee commodity chain, Rowman and Littlefield, Oxford, UK (2014).

E. Tombari, G. Salvetti, C. Ferrari, G. P. Johari, Kinetics and Thermodynamics of Sucrose Hydrolysis from RealTime Enthalpy and Heat Capacity Measurements, Journal of Physical Chemistry B 111 (2007), 496-501.

M. A. J. S. van Boekel, Formation of flavour compounds in the Maillard reaction, Biotechnology Advances 24 (2006), 230-233.

X. Wang, L. T. Lim, A kinetics and modeling study of coffee roasting under isothermal conditions, Food and Bioprocess Technology 7 (2014), 621-632.

M. Wang, N. Pan, Predictions of effective physical properties of complex multiphase materials, Materials Science and Engineering R 63 (2008), 1-30.

J. Zhang, A.K. Datta, Mathematical modeling of bread baking process, Journal of Food Engineering 75 (2006), 78-89. 